\title{
Bounding the Efficiency of RoAd Pricing
}

\author{
Hai Yang* and Wei Xu \\ Department of Civil Engineering, The Hong Kong University of Science \& Technology \\ Clear Water Bay, Kowloon, Hong Kong, P.R. China \\ and \\ Benjamin Heydecker \\ Centre for Transport Studies, University College London \\ Gower Street, London, WC1E, 6BT, England
}

\begin{abstract}
This paper deals with the following question associated with congestion pricing in a general network with either fixed or elastic travel demand: what is the maximum efficiency loss of a general second-best pricing scheme due to inexact marginal-cost pricing in comparison with the first-best pricing or system optimum case? A formal answer to this question is provided by establishing an inefficiency bound associated with a given road pricing scheme. An application of the methods is provided for the practical trial-and-error implementation of marginal-cost pricing with unknown demand functions.
\end{abstract}

Keywords: Nash game, inefficiency, congestion pricing, traffic equilibria

\section{INTRODUCTION}

Roadway congestion is a source of enormous economic costs. In principle, many of these costs can be prevented, as they result from socially inefficient choices by individual drivers. A number of regions have considered alleviating roadway congestion by introducing congestion pricing. Indeed, road pricing has become one of the priorities on transport policy agendas throughout the world. An increasing number of congestion pricing schemes have been proposed, tested or implemented worldwide. Examples include the US's value pricing scheme, recent EU green and white papers, Dutch initiatives, electronic road pricing schemes

\footnotetext{
* Corresponding author. Tel.: +852-2358-7178; Fax:+852-2358-1534, E-mail address: cehyang@ust.hk (H. Yang)
} 
in Singapore and Hong Kong, and the London congestion charging scheme that was introduced in February 2003. It is increasingly believed that road pricing may offer an effective instrument to manage travel demand, and to raise revenue that may, for instance, be used for transport improvements, and a new generation of road-use pricing technologies will be widely considered for introduction on many congested road networks.

From a theoretical perspective, road pricing has been a subject of substantial researches for a few decades by transportation economists and scientists. The initial idea of road pricing was put forward by Pigou (1920), who used the example of a congested road to make points on externalities and optimal congestion charges. Seminal works on both intellectual and practical developments after Pigou's idea include Walters (1961), Beckmann (1965), Vickrey (1969), Dafermos and Sparrow (1971), Dafermos (1973) and Smith (1979a). The key objective of road pricing is to achieve an optimal flow distribution pattern, which minimizes the total network travel time in the case of fixed demand, or maximize the total economic benefit in the case of elastic demand, by charging users on congested links in the network. It is widely recognized that the System Optimum (SO) can be decentralized as a Wardropian User Equilibrium (UE) (Wardrop, 1952), by charging tolls on all links according to the principle of marginal-cost or first-best pricing. The optimal toll on a road link is equal to the difference between the marginal social cost and the marginal private cost, which can internalize the user externalities and thus achieve a SO flow pattern in the network (Yang and Huang, 1998). It is also recognized that, in spite of its perfect theoretical basis, the first-best pricing scheme is of little practical interest. The problem stems from the fact that it is impractical to charge users on each network link in view of the operating cost and public acceptance. Due to the imperfection of the first-best pricing from a political and technical implementation perspective, the second-best charging schemes are more practically relevant, and indeed have received ample attention recently. A wide variety of second-best pricing schemes in general networks are developed to determine the optimal tolls for system performance optimization, under given physical and economic pricing constraints. Readers are suggested to refer to Yang and Huang (2005) and Lawphongpanich et al. (2006) for recent comprehensive treatments of the first-best and second-best pricing problems in general networks.

Among the various social, economic and technical issues associated with congestion pricing in general traffic networks, the following question is of particular interest here: what is the maximum efficiency loss of a general second-best pricing scheme due to inexact marginalcost pricing in comparison with the first-best pricing or system optimum case? Clearly, a formal answer to this question should prove to be very important and meaningful for design and evaluation of actual pricing schemes in the presence of pricing constraints. 
In this paper we make a full investigation of the posted questions in the spirit of "price of anarchy', a term that was first dubbed and used recently for bounding the inefficiency of equilibria in non-atomic congestion games (Papadimitriou, 2001). The price of anarchy for a non-atomic congestion game is determined by looking for the worst possible ratio between the total cost incurred by players in an equilibrium situation and in an outcome of minimumpossible total cost or system optimum; it has been examined by, for example, Roughgarden (2003), Chau and Sim (2003), Roughgarden and Tardos (2004), Perakis (2004), and Correa et al. (2004, 2005). It is suggested that readers refer to Roughgarden (2005) for a recent comprehensive review of this emerging research subject.

The paper is organized below. In next section, we present our bounding results of the efficiency loss in terms of total system travel time minimization for an arbitrary congestion pricing scheme with fixed demand; we show that our new bounding formula can generate the existing results of price of anarchy for self-routing in the special case of zero pricing, and lead to zero efficiency loss or maximum efficiency gain in the special case of first-best pricing. In Section 3, we extend the bounding methods to the general congestion pricing scheme with elastic demand using social welfare as a system performance measure. In Section 4, we propose and discuss briefly an alternative bounding approach to the problems of interest. In Section 5, we provide an application of the proposed efficiency bounding methods for the practical trial-and-error implementation of the congestion pricing problem with unknown demand functions. General conclusions and suggestions for further researches are given in Section 6.

\section{EFFICIENCY GAIN AND Loss Of PRICING WITH FIXED DEMAND}

\subsection{Traffic Equilibria and Inefficiency Measure}

Let $G(N, A)$ denote a transportation network, with a set of nodes $N$ and a set of links $A$, together with a set of Origin-Destination (OD) pairs $W$. Let $R_{w}$ denote the set of all simple paths connecting OD pair $w \in W, d_{w}\left(d_{w}>0\right)$ the demand for travel of users between OD pair $w \in W$, and $f_{r w}$ the flow of users on path $r \in R_{w}$. For given OD demands, the feasible set, $\Omega_{v}$, of link flow vector, $v=\left(v_{a}: a \in A\right)^{\mathrm{T}}$, with ' $\mathrm{T}$ ' denoting transpose of a vector, can be expressed as follows:

$$
\Omega_{v}=\left\{\left(v_{a}: a \in A\right) \mid v_{a}=\sum_{w \in W} \sum_{r \in R_{w}} f_{r w} \delta_{a r}, a \in A, \sum_{r \in R_{w}} f_{r w}=d_{w}, f_{r w} \geq 0, r \in R_{w}, w \in W\right\}
$$

where $\delta_{a r}=1$ if route $r$ uses link $a$ and 0 otherwise. Let $t_{a}$ denote the travel time on 
link $a \in A$. For simplicity, $t_{a}=t_{a}\left(v_{a}\right)$ is assumed to be a strictly increasing, convex and differentiable function of flow $v_{a}$ on link $a$ only (separable case), i.e., $\mathrm{d} t_{a}\left(v_{a}\right) / \mathrm{d} v_{a}>0$ and $\mathrm{d}^{2} t_{a}\left(v_{a}\right) / \mathrm{d} v_{a}^{2} \geq 0, a \in A$.

Consider a general pricing scheme, $u=\left(u_{a}: a \in A\right)^{\mathrm{T}} \geq 0$, where $u_{a}$ is the toll charge on link $a \in A$ in equivalent time unit. Let $v^{\text {ue }}=v^{\text {ue }}(u)$ be the corresponding UE link flow vector associated with pricing scheme $u$. It is well known that $v^{\text {ue }}$ is the solution to the following standard UE program with fixed demands and separable link cost functions (Sheffi, 1985):

$$
v^{\mathrm{ue}}=v^{\mathrm{ue}}(u)=\arg \min _{v \in \Omega_{v}} \sum_{a \in A} \int_{0}^{v_{a}}\left\{t_{a}(\omega)+u_{a}\right\} \mathrm{d} \omega
$$

Note that hereinafter, for simplicity, $v^{\text {ue }}$ always denotes $v^{\text {ue }}(u)$, the UE link flow associated with link toll charge $u$ under consideration, the un-tolled UE link flow corresponds to $v^{\text {ue }}(u)$ at $u=0$.

Alternatively, the UE problem can be formulated as the following equivalent Variational Inequalities (VI) in terms of link flow variables (Smith, 1979b; Dafermos, 1980). Find $v^{\text {ue }} \in \Omega_{v}$ such that

$$
\left(t\left(v^{\text {ue }}\right)+u\right)^{\mathrm{T}}\left(v-v^{\text {ue }}\right) \geq 0, \text { for any } v \in \Omega_{v}
$$

It is known that if $t_{a}\left(v_{a}\right)$ is continuous and monotonically increasing in $v_{a}$, then there exists a unique link flow solution, $v^{\text {ue }}(u)$, for given pricing scheme $u \geq 0$. Then the total system travel time at UE under a pricing scheme $u$ is given by

$$
T\left(v^{\mathrm{ue}}(u)\right)=\sum_{a \in A} t_{a}\left(v_{a}^{\mathrm{ue}}(u)\right) v_{a}^{\mathrm{ue}}(u)
$$

On the other hand, the standard SO model that minimizes the total system travel time is given by

$$
\min _{v \in \Omega_{v}} T(v)=\sum_{a \in A} t_{a}\left(v_{a}\right) v_{a}
$$

If $t_{a}\left(v_{a}\right)$ is monotonically increasing and convex in $v_{a}$, then $t_{a}\left(v_{a}\right) v_{a}$ is strictly convex and hence the solution to the SO problem (5) is unique. Let $v^{\text {so }}$ denote the unique link flow solution of the SO problem, the corresponding minimum system travel time associated with SO link flows is denoted by $T\left(v^{\mathrm{so}}\right)$.

For a given general pricing scheme $u$, we define the ratios: 


$$
\begin{aligned}
\rho_{\mathrm{fd}}^{\mathrm{ue}}(u) & =\frac{T\left(v^{\mathrm{ue}}(u)\right)}{T\left(v^{\mathrm{so}}\right)} \\
\phi_{\mathrm{fd}}^{\mathrm{ue}}(u) & =\frac{T\left(v^{\mathrm{ue}}(u)\right)-T\left(v^{\mathrm{so}}\right)}{T\left(v^{\mathrm{so}}\right)}=\rho_{\mathrm{fd}}^{\mathrm{ue}}(u)-1
\end{aligned}
$$

where ' $\mathrm{fd}$ ' denotes the case of fixed demand. Clearly, $\rho_{\mathrm{fd}}^{\mathrm{ue}}(u) \geq 1$, and $\phi_{\mathrm{fd}}^{\mathrm{ue}}(u) \geq 0$. The latter measure, as a result of $\rho_{\mathrm{fd}}^{\mathrm{ue}}(u)$, represents the relative gap in system performance measure of total travel time between the UE flow pattern under the current second-best pricing scheme $u$ and the system-optimal one.

As already pointed out, a first-best marginal-cost pricing scheme can drive a UE flow pattern to the system optimum. In other words, $T\left(v^{\mathrm{ue}}(u)\right)_{u=u^{\mathrm{mc}}}=T\left(v^{\mathrm{so}}\right)$ and $\phi_{\mathrm{fd}}^{\mathrm{ue}}(u)_{u=u^{\mathrm{mc}}}=0$, where $u^{\mathrm{mc}}$ denotes the first-best marginal-cost pricing link toll vector:

$$
u_{a}^{\mathrm{mc}}=v_{a}^{\mathrm{so}} t_{a}^{\prime}\left(v_{a}^{\mathrm{so}}\right), \forall a \in A
$$

where $t_{a}^{\prime}\left(v_{a}\right)=\mathrm{d} t_{a}\left(v_{a}\right) / \mathrm{d} v_{a}$ denotes the derivative of link cost function in link flow. Therefore, one may regard $\phi_{\mathrm{fd}}^{\text {ue }}(u)$ as the relative efficiency loss associated with a general second-best pricing scheme $u$ in comparison with the first-best one $u^{\text {mc }}$. This efficiency loss also determines the room or potential for further improvement of the second-best pricing scheme per se.

If $u=0$, then the ratio $\rho_{\mathrm{fd}}^{\mathrm{ue}}(u)_{u=0}$ in (6) becomes the measure of the inefficiency or price of anarchy of the selfish user equilibria (Roughgarden, 2005). This ratio can also be regarded as the efficiency gain of a marginal-cost pricing scheme, because, as already mentioned, it can drive a UE flow pattern to the system optimal one, or completely remove the inefficiency of selfish user equilibria.

\subsection{Bound for Efficiency Gain and Loss}

Our purpose here is to find an upper bound of $\rho_{\mathrm{fd}}^{\mathrm{ue}}(u)$ for a given pricing scheme $u$, thereby quantifying the maximum efficiency loss of the second-best pricing scheme $u$. As a special case, such a bound will naturally give the maximum efficiency gain that can be achieved through a first-best pricing scheme in comparison with the non-tolling case, as just mentioned. In a similar spirit of Correa et al. (2005) who considered inefficiency bound in the absence of toll pricing, from VI (3) we have

$$
T\left(v^{\text {ue }}\right) \leq \sum_{a \in A} t_{a}\left(v_{a}\right) v_{a}+\sum_{a \in A}\left(t_{a}\left(v_{a}^{\text {ue }}\right)-t_{a}\left(v_{a}\right)\right) v_{a}+\sum_{a \in A}\left(v_{a}-v_{a}^{\text {ue }}\right) u_{a}, \forall v \in \Omega_{v}
$$


For each link cost function $t_{a}=t_{a}\left(v_{a}\right)$ and nonnegative UE link flow $v_{a}^{\text {ue }} \geq 0$, we define the following parameter for each link $a \in A$, associated with link toll charge $u_{a}$ :

$$
\begin{aligned}
& \gamma_{a}\left(t_{a}, v_{a}^{\mathrm{ue}}, u_{a}\right)=\max _{v_{a} \geq 0} \frac{\left(t_{a}\left(v_{a}^{\mathrm{ue}}\right)-t_{a}\left(v_{a}\right)\right) v_{a}+\left(v_{a}-v_{a}^{\mathrm{ue}}\right) u_{a}}{t_{a}\left(v_{a}^{\mathrm{ue}}\right) v_{a}^{\mathrm{ue}}}, \text { if } 0 \leq u_{a} \leq v_{a}^{\mathrm{ue}} t_{a}^{\prime}\left(v_{a}^{\mathrm{ue}}\right) \\
& \gamma_{a}\left(t_{a}, v_{a}^{\mathrm{ue}}, u_{a}\right)=\max _{v_{a} \geq 0} \frac{\left(t_{a}\left(v_{a}^{\mathrm{ue}}\right)-t_{a}\left(v_{a}\right)\right) v_{a}+\left(v_{a}-v_{a}^{\mathrm{ue}}\right) u_{a}}{t_{a}\left(v_{a}\right) v_{a}}, \text { if } u_{a} \geq v_{a}^{\mathrm{ue}} t_{a}^{\prime}\left(v_{a}^{\mathrm{ue}}\right)
\end{aligned}
$$

Here, $0 / 0=0$ by convention, the reason for using the two different definitions (10) and (11) is given later in Remark 2.

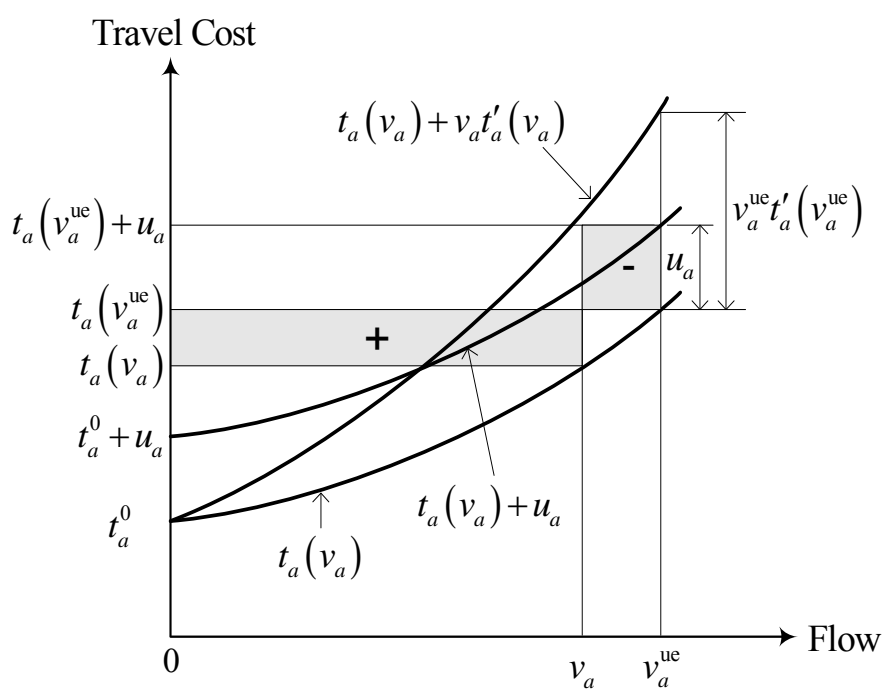

Figure 1. Geometric illustration of the numerator of eqn. (10) for $0 \leq u_{a} \leq v_{a}^{\mathrm{ue}} t_{a}^{\prime}\left(v_{a}^{\mathrm{ue}}\right)$

We first consider the case of $0 \leq u_{a} \leq v_{a}^{\mathrm{ue}} t_{a}^{\prime}\left(v_{a}^{\mathrm{ue}}\right)$. Because the denominator in (10) is fixed, our task here is to solve the following one-dimensional maximization problem for $v_{a}$ :

$$
\max _{v_{a} \geq 0} F_{1}\left(v_{a}\right)=\left(t_{a}\left(v_{a}^{\mathrm{ue}}\right)-t_{a}\left(v_{a}\right)\right) v_{a}+\left(v_{a}-v_{a}^{\mathrm{ue}}\right) u_{a}
$$

Note that it is assumed that $t_{a}\left(v_{a}\right)$ is monotonically increasing and convex and hence $F_{1}\left(v_{a}\right)$ is strictly concave. Figures 1 plots the area corresponding to the numerator of eqn. (10) for $0 \leq u_{a} \leq v_{a}^{\text {ue }} t_{a}^{\prime}\left(v_{a}^{\text {ue }}\right)$, where the term, $\left(t_{a}\left(v_{a}^{\text {ue }}\right)-t_{a}\left(v_{a}\right)\right) v_{a}$, has a positive value corresponding to the area of the rectangle denoted by ' + ' and the term, $\left(v_{a}-v_{a}^{\text {ue }}\right) u_{a}$, has a negative value corresponding to the area of the rectangle denoted by '-'. Let $\mathrm{d} F_{1}\left(v_{a}\right) / \mathrm{d} v_{a}=0$, we have

$$
t_{a}\left(v_{a}^{*}\right)+v_{a}^{*} t_{a}^{\prime}\left(v_{a}^{*}\right)=t_{a}\left(v_{a}^{\mathrm{ue}}\right)+u_{a}
$$


As illustrated in Figure 2, a unique solution $0 \leq v_{a}^{*} \leq v_{a}^{\mathrm{ue}}$ is obtained for $0 \leq u_{a} \leq v_{a}^{\mathrm{ue}} t_{a}^{\prime}\left(v_{a}^{\mathrm{ue}}\right)$, because the left hand side of eqn. (13) is a strictly increasing function of $v_{a}$ for increasing and convex $t_{a}\left(v_{a}\right)$, and the right hand side is less than or equal to $t_{a}\left(v_{a}^{\mathrm{ue}}\right)+v_{a}^{\mathrm{ue}} t_{a}^{\prime}\left(v_{a}^{\mathrm{ue}}\right)$.

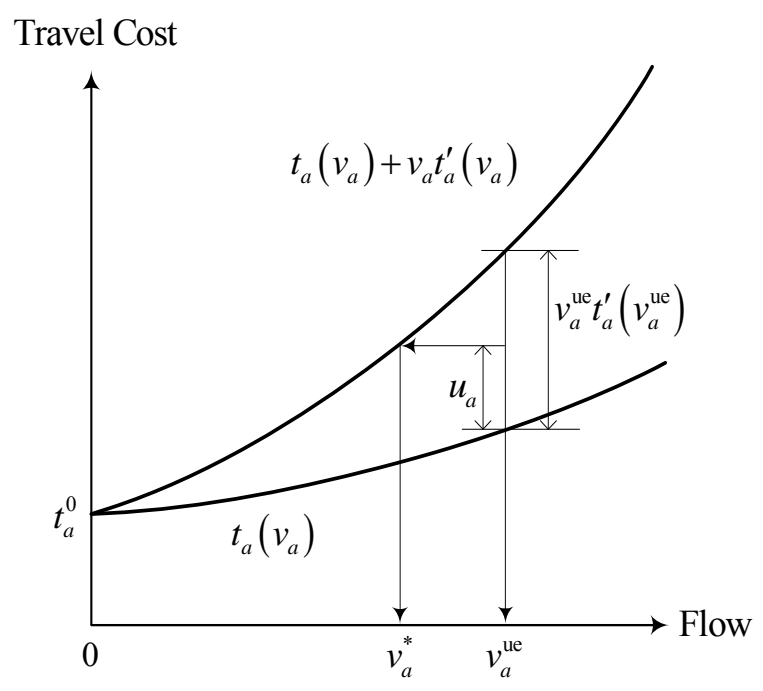

Figure 2. Geometric illustration of the solution to equation (13)

Next, we briefly examine the case of $u_{a} \geq v_{a}^{\text {ue }} t_{a}^{\prime}\left(v_{a}^{\text {ue }}\right)$. Define

$$
F_{2}\left(v_{a}\right)=\frac{\left(t_{a}\left(v_{a}^{\mathrm{ue}}\right)-t_{a}\left(v_{a}\right)\right) v_{a}+\left(v_{a}-v_{a}^{\mathrm{ue}}\right) u_{a}}{t_{a}\left(v_{a}\right) v_{a}}=-1+\frac{\left(t_{a}\left(v_{a}^{\mathrm{ue}}\right)+u_{a}\right) v_{a}-v_{a}^{\mathrm{ue}} u_{a}}{t_{a}\left(v_{a}\right) v_{a}}
$$

Clearly, this function is continuously differentiable for $v_{a}>0$ and has the properties that $\lim _{v_{a} \rightarrow+\infty} F_{2}\left(v_{a}\right)=-1, \lim _{v_{a} \rightarrow 0} F_{2}\left(v_{a}\right)=-\infty$ and $F_{2}\left(v_{a}^{\text {ue }}\right)=0$, a maximum point must exist in the interior of $(0,+\infty)$. Again let $\mathrm{d} F_{2}\left(v_{a}\right) / \mathrm{d} v_{a}=0$, we have

$$
\left[t_{a}\left(v_{a}^{\mathrm{ue}}\right)+u_{a}\right] v_{a} t_{a}\left(v_{a}\right)-\left[\left(t_{a}\left(v_{a}^{\mathrm{ue}}\right)+u_{a}\right) v_{a}-v_{a}^{\mathrm{ue}} u_{a}\right]\left(t_{a}\left(v_{a}\right)+v_{a} t_{a}^{\prime}\left(v_{a}\right)\right)=0
$$

It simplifies into:

$$
\frac{t_{a}\left(v_{a}^{\mathrm{ue}}\right)+u_{a}}{v_{a}^{\mathrm{ue}} u_{a}}=\frac{t_{a}\left(v_{a}\right)+v_{a} t_{a}^{\prime}\left(v_{a}\right)}{\left(v_{a}\right)^{2} t_{a}^{\prime}\left(v_{a}\right)}
$$

In addition, the derivative of the right hand side in (15) is

$$
\mathrm{d}\left(\frac{t_{a}\left(v_{a}\right)+v_{a} t_{a}^{\prime}\left(v_{a}\right)}{\left(v_{a}\right)^{2} t_{a}^{\prime}\left(v_{a}\right)}\right) / \mathrm{d} v_{a}=\frac{-2 v_{a} t_{a}\left(v_{a}\right) t_{a}^{\prime}\left(v_{a}\right)-\left(v_{a}\right)^{2} t_{a}\left(v_{a}\right) t_{a}^{\prime \prime}\left(v_{a}\right)}{\left[\left(v_{a}\right)^{2} t_{a}^{\prime}\left(v_{a}\right)\right]^{2}}<0
$$

There is a unique solution to eqn. (15). Thus one can conclude that there exists one unique 
positive solution $v_{a}^{*}$ for maximizing $F_{2}\left(v_{a}\right)$. For illustration, Figure 3 plots the curves of $F_{2}\left(v_{a}\right)$ for $t(v)=6.0+0.9(v / 200)^{p}$ with $u=9, v^{\text {ue }}=85$ and $p=1,2,3,4$. It is clear that there exists a unique maximum point for each curve.

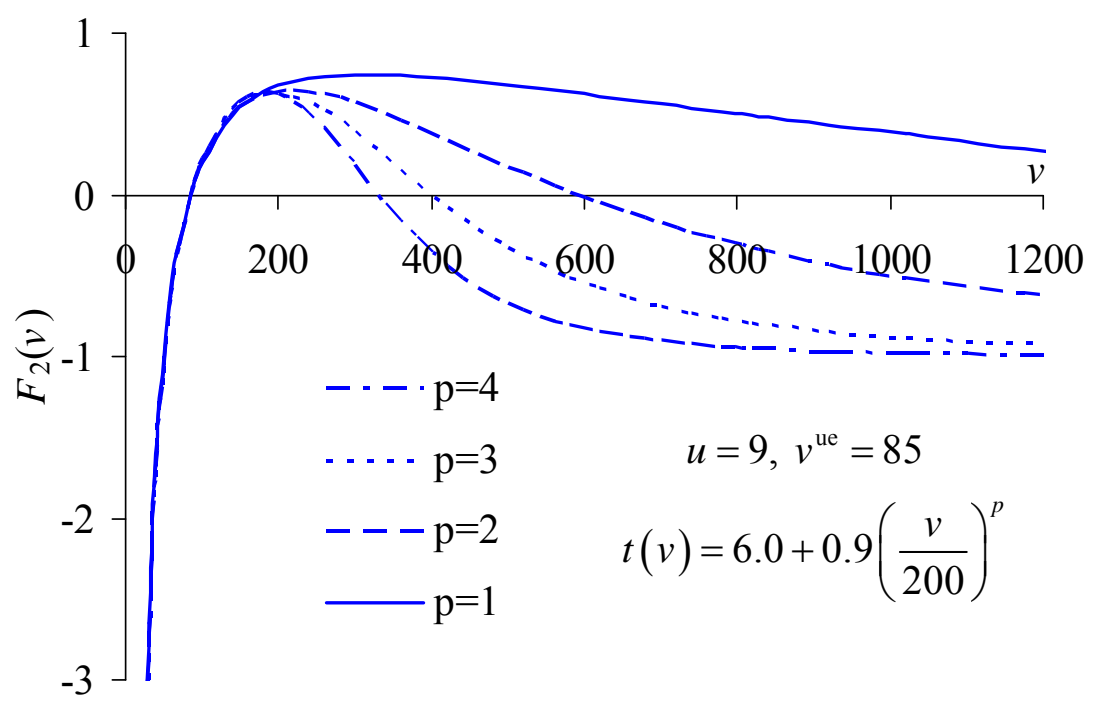

Figure 3. The general shape and maximum of function (14)

For a given class $\mathcal{C}$ of link cost functions (for example, a family of linear cost functions or polynomials of a certain degree) and a pricing scheme $u$ with $v^{\text {ue }}=v^{\text {ue }}(u)$, we define

$$
\gamma(\mathcal{C}, u)=\max _{t_{a} \in \mathcal{C}, a \in A} \gamma_{a}\left(t_{a}, v_{a}^{\mathrm{ue}}, u_{a}\right)
$$

With this definition, the following theorem follows immediately.

Theorem 1 Let $v^{\text {ue }}=v^{\text {ue }}(u)$ be a UE link flow pattern associated with a pricing scheme $u$, with separable link cost functions drawn from a given class $\mathcal{C}$, and let $v^{\mathrm{so}}$ be an SO link flow solution, then

$$
\begin{aligned}
& T\left(v^{\mathrm{ue}}(u)\right) \leq \frac{1}{1-\gamma(\mathcal{C}, u)} T\left(v^{\mathrm{so}}\right) \quad \text { if } \quad 0 \leq u_{a} \leq v_{a}^{\mathrm{ue}} t_{a}^{\prime}\left(v_{a}^{\mathrm{ue}}\right), \forall a \in A \\
& T\left(v^{\mathrm{ue}}(u)\right) \leq(1+\gamma(\mathcal{C}, u)) T\left(v^{\mathrm{so}}\right) \quad \text { if } u_{a} \geq v_{a}^{\mathrm{ue}} t_{a}^{\prime}\left(v_{a}^{\mathrm{ue}}\right), \forall a \in A
\end{aligned}
$$

Proof: The proof is similar to Correa et al. (2005) in the absence of toll pricing. We consider the case of $0 \leq u_{a} \leq v_{a}^{\text {ue }} t_{a}^{\prime}\left(v_{a}^{\text {ue }}\right), \forall a \in A$. A similar proof applies for $u_{a} \geq v_{a}^{\mathrm{ue}} t_{a}^{\prime}\left(v_{a}^{\mathrm{ue}}\right), \forall a \in A$. From definitions (10) and (16), we have 


$$
\begin{aligned}
\sum_{a \in A}\left(t_{a}\left(v_{a}^{\mathrm{ue}}\right)-t_{a}\left(v_{a}\right)\right) v_{a}+\left(v_{a}-v_{a}^{\mathrm{ue}}\right) u_{a} & \leq \sum_{a \in A} \gamma_{a}\left(t_{a}, v_{a}^{\mathrm{ue}}, u_{a}\right) t_{a}\left(v_{a}^{\mathrm{ue}}\right) v_{a}^{\mathrm{ue}} \\
& \leq \sum_{a \in A} \gamma(\mathcal{C}, u) t_{a}\left(v_{a}^{\mathrm{ue}}\right) v_{a}^{\mathrm{ue}}=\gamma(\mathcal{C}, u) T\left(v^{\mathrm{ue}}\right)
\end{aligned}
$$

Substituting (19) into (9) yields $T\left(v^{\text {ue }}\right) \leq \sum_{a \in A} t_{a}\left(v_{a}\right) v_{a}+\gamma(\mathcal{C}, u) T\left(v^{\text {ue }}\right), \forall v \in \Omega_{v}$. Let $v=v^{\text {so }}$, we have (17).

Theorem 1 simply states that, for the UE problem with fixed demand under a pricing scheme $u$, we have:

(i) if $0 \leq u_{a} \leq v_{a}^{\text {ue }} t_{a}^{\prime}\left(v_{a}^{\text {ue }}\right), \forall a \in A$

$$
\begin{aligned}
& \rho_{\mathrm{fd}}^{\mathrm{ue}}(u) \leq \frac{1}{1-\gamma(\mathcal{C}, u)} \\
& \phi_{\mathrm{fd}}^{\mathrm{ue}}(u)=\rho_{\mathrm{fd}}^{\mathrm{ue}}(u)-1 \leq \frac{\gamma(\mathcal{C}, u)}{1-\gamma(\mathcal{C}, u)}
\end{aligned}
$$

(ii) if $u_{a} \geq v_{a}^{\text {ue }} t_{a}^{\prime}\left(v_{a}^{\text {ue }}\right), \forall a \in A$

$$
\begin{aligned}
& \rho_{\mathrm{fd}}^{\mathrm{ue}}(u) \leq 1+\gamma(\mathcal{C}, u) \\
& \phi_{\mathrm{fd}}^{\mathrm{ue}}(u)=\rho_{\mathrm{fd}}^{\mathrm{ue}}(u)-1 \leq \gamma(\mathcal{C}, u)
\end{aligned}
$$

Remark 1 In the special case of marginal-cost pricing, we have $u_{a}=v_{a}^{\mathrm{ue}} t_{a}^{\prime}\left(v_{a}^{\mathrm{ue}}\right)$ for all $a \in A$. Then, it follows that $v_{a}^{*}=v_{a}^{\mathrm{ue}}$ from (13), and $\gamma_{a}\left(t_{a}, v_{a}^{\mathrm{ue}}, u_{a}\right)=0$ from eqn. (10). As a result, $\gamma(\mathcal{C}, u)=0, \rho_{\mathrm{fd}}^{\mathrm{ue}}(u)=1$ and $\phi_{\mathrm{fd}}^{\mathrm{ue}}(u)=0$, which simply implies that there is no efficiency loss in the system under a marginal-cost pricing scheme. Similarly, we can apply eqn. (11) for $u_{a}=v_{a}^{\text {ue }} t_{a}^{\prime}\left(v_{a}^{\text {ue }}\right), a \in A$. In this case, from eqn. (15) we have a unique solution of the equation given by $v_{a}^{*}=v_{a}^{\mathrm{ue}}, a \in A$. Then, from eqn. (11), $\gamma_{a}\left(t_{a}, v_{a}^{\mathrm{ue}}, u_{a}\right)=0$. We thus obtain the same results: $\gamma(\mathcal{C}, u)=0, \rho_{\mathrm{fd}}^{\text {ue }}(u)=1$ and $\phi_{\mathrm{fd}}^{\text {ue }}(u)=0$.

Remark 2 We have adopted two different definitions of $\gamma_{a}\left(t_{a}, v_{a}^{\text {ue }}, u_{a}\right)$ given in (10) and (11) respectively for the two cases of $0 \leq u_{a} \leq v_{a}^{\mathrm{ue}} t_{a}^{\prime}\left(v_{a}^{\mathrm{ue}}\right)$ and $u_{a} \geq v_{a}^{\mathrm{ue}} t_{a}^{\prime}\left(v_{a}^{\mathrm{ue}}\right)$, which, as pointed out in Remark 1, give rise to consistent values of $\gamma_{a}\left(t_{a}, v_{a}^{\text {ue }}, u_{a}\right)=0$ at a marginalcost pricing scheme: $u_{a}=v_{a}^{\mathrm{ue}} t_{a}^{\prime}\left(v_{a}^{\mathrm{ue}}\right), a \in A$. Two separate definitions for the two cases are necessary, otherwise, the bound may become meaningless. First, from (20) or (17), the bound is meaningful only if $0 \leq \gamma(\mathcal{C}, u) \leq 1$, which indeed holds for the current definition 
(10) for the following simple reason. As already mentioned, for $0 \leq u_{a} \leq v_{a}^{\mathrm{ue}} t_{a}^{\prime}\left(v_{a}^{\mathrm{ue}}\right)$, we have $0 \leq v_{a}^{*} \leq v_{a}^{\text {ue }}$, thus $\left(v_{a}-v_{a}^{\text {ue }}\right) u_{a} \leq 0$ and $\left(t_{a}\left(v_{a}^{\text {ue }}\right)-t_{a}\left(v_{a}\right)\right) v_{a} \leq t_{a}\left(v_{a}^{\text {ue }}\right) v_{a}^{\text {ue }}$. This implies that $F_{1}\left(v_{a}\right) \leq t_{a}\left(v_{a}^{\mathrm{ue}}\right) v_{a}^{\mathrm{ue}}$ for $0 \leq v_{a} \leq v_{a}^{\mathrm{ue}}$. As a result, $0 \leq \max _{v_{a} \geq 0} F_{1}\left(v_{a}\right) \leq t_{a}\left(v_{a}^{\mathrm{ue}}\right) v_{a}^{\mathrm{ue}}$ in (12) or $0 \leq \gamma_{a}\left(t_{a}, v_{a}^{\text {ue }}, u_{a}\right) \leq 1$. If, however, we extend definition (10) to the case of $u_{a} \geq v_{a}^{\mathrm{ue}} t_{a}^{\prime}\left(v_{a}^{\mathrm{ue}}\right)$, then it is possible that $\gamma_{a}\left(t_{a}, v_{a}^{\text {ue }}, u_{a}\right)>1$ when $u_{a}$ is sufficiently large. To see this, we can choose a constant $v_{a}$ slightly greater than $v_{a}^{\mathrm{ue}}$, then the term $\left(v_{a}-v_{a}^{\mathrm{ue}}\right) u_{a}$ in (10) is positive and always increases with $u_{a}$. Because the negative term $\left(t_{a}\left(v_{a}^{\mathrm{ue}}\right)-t_{a}\left(v_{a}\right)\right) v_{a}$ is bounded (as $v_{a}$ is upper-bounded by, for example, total traffic demand) and $t_{a}\left(v_{a}^{\text {ue }}\right) v_{a}^{\text {ue }}$ is a positive constant in (10), $\gamma_{a}\left(t_{a}, v_{a}^{\text {ue }}, u_{a}\right)$ becomes much greater than 1 as $u_{a}$ becomes large. Second, from (18) or (22), $\gamma(\mathcal{C}, u)$ should not always go to positive infinity for meaningful $u$, otherwise, the presented bound becomes ineffective. This is precisely fulfilled by the definition of $\gamma_{a}\left(t_{a}, v_{a}^{\mathrm{ue}}, u_{a}\right)$ in (11) for $u_{a} \geq v_{a}^{\mathrm{ue}} t_{a}^{\prime}\left(v_{a}^{\mathrm{ue}}\right)$. On the contrary, if we extend definition (11) to the case of $0 \leq u_{a} \leq v_{a}^{\text {ue }} t_{a}^{\prime}\left(v_{a}^{\text {ue }}\right)$, then the value of $\gamma_{a}\left(t_{a}, v_{a}^{\mathrm{ue}}, u_{a}\right)$ may become too large to be a sensible bound if $u_{a}$ is sufficiently small. This can be seen in the extreme case of $u_{a}=0$ and zero free-flow travel time, because $\gamma_{a}\left(t_{a}, v_{a}^{\mathrm{ue}}, u_{a}\right) \rightarrow+\infty$ as $v_{a} \rightarrow 0$ in this case.

Note that the bounding formulas (17) and (18) assume that the toll charge is either uniformly less (or greater) than the corresponding congestion externality for all links in the network. It is obviously unrealistic in practice. Toll charge for a link in a network can be either less or greater than the corresponding congestion externality in a second-best pricing scheme, such as the sequential experimental pricing scheme to be considered later. It is therefore necessary to establish an inefficiency bound for general second-best pricing schemes that can accommodate both possibilities. To do this, we now consider the following relaxation for the inefficiency bounding.

Let $A_{1}$ and $A_{2}$ be the link sets consisting of those links whose toll charges are less than and greater than their congestion externalities, respectively

$$
A_{1}=\left\{a \mid 0 \leq u_{a}<v_{a}^{\mathrm{ue}} t_{a}^{\prime}\left(v_{a}^{\mathrm{ue}}\right), a \in A\right\} ; A_{2}=\left\{a \mid u_{a}>v_{a}^{\mathrm{ue}} t_{a}^{\prime}\left(v_{a}^{\mathrm{ue}}\right), a \in A\right\}
$$

If $u_{a}=v_{a}^{\text {ue }} t_{a}^{\prime}\left(v_{a}^{\text {ue }}\right)$ then $a$ can be classified into either $A_{1}$ or $A_{2}$. With this classification, we denote 


$$
\begin{aligned}
& \gamma_{1}(\mathcal{C}, u)=\max _{t_{a} \in \mathcal{C}, a \in A_{1}} \gamma_{a}\left(t_{a}, v_{a}^{\mathrm{ue}}, u_{a}\right) \text { for } 0 \leq u_{a} \leq v_{a}^{\mathrm{ue}} t_{a}^{\prime}\left(v_{a}^{\mathrm{ue}}\right), a \in A_{1} \\
& \gamma_{2}(\mathcal{C}, u)=\max _{t_{a} \in \mathcal{C}, a \in A_{2}} \gamma_{a}\left(t_{a}, v_{a}^{\mathrm{ue}}, u_{a}\right) \text { for } u_{a} \geq v_{a}^{\mathrm{ue}} t_{a}^{\prime}\left(v_{a}^{\mathrm{ue}}\right), a \in A_{2}
\end{aligned}
$$

where $\gamma_{a}\left(t_{a}, v_{a}^{\text {ue }}, u_{a}\right)$ are defined respectively by (10) and (11) for $a \in A_{1}$ and $a \in A_{2}$. Then from the basic inequality (9), it follows that

$$
\begin{aligned}
T\left(v^{\mathrm{ue}}\right) & \leq \sum_{a \in A} t_{a}\left(v_{a}\right) v_{a}+\gamma_{1}(\mathcal{C}, u) \sum_{a \in A_{1}} t_{a}\left(v_{a}^{\mathrm{ue}}\right) v_{a}^{\mathrm{ue}}+\gamma_{2}(\mathcal{C}, u) \sum_{a \in A_{2}} t_{a}\left(v_{a}\right) v_{a} \\
& \leq \sum_{a \in A} t_{a}\left(v_{a}\right) v_{a}+\gamma_{1}(\mathcal{C}, u) T\left(v^{\mathrm{ue}}\right)+\gamma_{2}(\mathcal{C}, u) T(v)
\end{aligned}
$$

As before, using definition (6) and let $v_{a}=v_{a}^{\text {so }}$ in (27), we have

$$
T\left(v^{\mathrm{ue}}\right) \leq \frac{1+\gamma_{2}(\mathcal{C}, u)}{1-\gamma_{1}(\mathcal{C}, u)} T\left(v^{\mathrm{so}}\right)
$$

that is

$$
\rho_{\mathrm{fd}}^{\mathrm{ue}}(u) \leq \frac{1+\gamma_{2}(\mathcal{C}, u)}{1-\gamma_{1}(\mathcal{C}, u)}
$$

To sum up, we state the following Theorem.

Theorem 2 Let $v^{\text {ue }}=v^{\text {ue }}(u)$ be a UE link flow pattern associated with a pricing scheme $u$, with separable link cost functions drawn from a given class $\mathcal{C}$, and let $v^{\text {so }}$ be an SO link flow solution, then

$$
T\left(v^{\mathrm{ue}}(u)\right) \leq \frac{1+\gamma_{2}(\mathcal{C}, u)}{1-\gamma_{1}(\mathcal{C}, u)} T\left(v^{\mathrm{so}}\right)
$$

Note that the above general results in Theorem 2 depend on the specific instance, because they depend on the toll and the marginal cost in each link. We next look at the special case with a class of BPR (Bureau of Public Road) link cost functions and show how the bound result in this case can get ride of the dependence of the network topology.

\subsection{Bound with BPR Link Cost Functions}

For a given positive integer $p$, let $\mathcal{C}_{p}$ denote the set of the following convex BPR-type cost function that is widely used in road traffic assignment

$$
t_{a}\left(v_{a}\right)=t_{a}^{0}+\alpha_{a}\left(v_{a}\right)^{p}, a \in A
$$

where $t_{a}^{0} \geq 0$ and $\alpha_{a} \geq 0$.

For given toll charge $u_{a}, \quad 0 \leq u_{a} \leq v_{a}^{\text {ue }} t_{a}^{\prime}\left(v_{a}^{\text {ue }}\right), a \in A_{1}$, eqn. (13) with specific cost function 
(30) becomes

$$
t_{a}^{0}+\alpha_{a}\left(v_{a}^{*}\right)^{p}+v_{a}^{*} \alpha_{a} p\left(v_{a}^{*}\right)^{p-1}=t_{a}^{0}+\alpha_{a}\left(v_{a}^{\mathrm{ue}}\right)^{p}+u_{a}
$$

and

$$
v_{a}^{*}=\left(\frac{\alpha_{a}\left(v_{a}^{\mathrm{ue}}\right)^{p}+u_{a}}{\alpha_{a}(1+p)}\right)^{\frac{1}{p}}
$$

Let

$$
u_{a}=\kappa_{a}\left[v_{a}^{\text {ue }} t_{a}^{\prime}\left(v_{a}^{\text {ue }}\right)\right]=\kappa_{a} \alpha_{a} p\left(v_{a}^{\text {ue }}\right)^{p}
$$

where the term $v_{a}^{\text {ue }} t_{a}^{\prime}\left(v_{a}^{\text {ue }}\right)$ is the link congestion externality at UE link flow $v_{a}^{\text {ue }}$ and $\kappa_{a}$ is a link-specific proportionality constant. With eqn. (33), eqn. (32) is simplified into

$$
v_{a}^{*}=\left(\frac{\left(1+\kappa_{a} p\right)}{(1+p)}\right)^{\frac{1}{p}} v_{a}^{\text {ue }}
$$

where $0 \leq \kappa_{a} \leq 1, a \in A_{1}$. Thus, it follows from (10) that

$$
\begin{aligned}
& \gamma_{a}\left(t_{a}, v_{a}^{\mathrm{ue}}, u_{a}\right)=\frac{\left(t_{a}\left(v_{a}^{\mathrm{ue}}\right)-t_{a}\left(v_{a}^{*}\right)\right) v_{a}^{*}+\left(v_{a}^{*}-v_{a}^{\mathrm{ue}}\right) u_{a}}{t_{a}\left(v_{a}^{\mathrm{ue}}\right) v_{a}^{\mathrm{ue}}} \\
& =\frac{\left(1-\frac{\left(1+\kappa_{a} p\right)}{(1+p)}\right) \alpha_{a}\left(v_{a}^{\mathrm{ue}}\right)^{p}\left(\left(\frac{\left(1+\kappa_{a} p\right)}{(1+p)}\right)^{\frac{1}{p}} v_{a}^{\mathrm{ue}}\right)+\left(\left(\frac{\left(1+\kappa_{a} p\right)}{(1+p)}\right)^{\frac{1}{p}}-1\right) v_{a}^{\mathrm{ue}} \kappa_{a} \alpha_{a} p\left(v_{a}^{\mathrm{ue}}\right)^{p}}{v_{a}^{\mathrm{ue}}\left(t_{a}^{0}+\alpha_{a}\left(v_{a}^{\mathrm{ue}}\right)^{p}\right)} \\
& =\frac{\frac{\left(1+\kappa_{a} p\right) p}{1+p}\left(\frac{\left(1+\kappa_{a} p\right)}{(1+p)}\right)^{\frac{1}{p}}-\kappa_{a} p}{t_{a}^{0}+\alpha_{a}\left(v_{a}^{\mathrm{ue}}\right)^{p}} \alpha_{a}\left(v_{a}^{\mathrm{ue}}\right)^{p}
\end{aligned}
$$

and

$$
\begin{aligned}
\gamma_{1}\left(\mathcal{C}_{p}, u\right) & =\max _{t_{a} \in \mathcal{C}_{p}, a \in A_{1}} \gamma_{a}\left(t_{a}, v_{a}^{\mathrm{ue}}, u_{a}\right) \\
& =\max _{t_{a} \in \mathcal{C}_{p}, a \in A_{1}} \frac{\frac{\left(1+\kappa_{a} p\right) p}{1+p}\left(\frac{\left(1+\kappa_{a} p\right)}{(1+p)}\right)^{\frac{1}{p}}-\kappa_{a} p}{t_{a}^{0}+\alpha_{a}\left(v_{a}^{\mathrm{ue}}\right)^{p}} \alpha_{a}\left(v_{a}^{\mathrm{ue}}\right)^{p} \\
\leq & \max _{a \in A_{1}} \frac{\left(1+\kappa_{a} p\right) p}{1+p}\left(\frac{\left(1+\kappa_{a} p\right)}{(1+p)}\right)^{\frac{1}{p}}-\kappa_{a} p
\end{aligned}
$$

because $t_{a}^{0} \geq 0$ for all $a \in A$. Note that $t_{a} \in \mathcal{C}_{p}$ is dropped in (35) because the term to be maximized becomes independent of specific cost functions in the class with the same number 
of power $p$.

Next we consider the case where $u_{a} \geq v_{a}^{\text {ue }} t_{a}^{\prime}\left(v_{a}^{\text {ue }}\right)$ or $\kappa_{a} \geq 1, a \in A_{2}$. By definition (11), we have

$$
\begin{aligned}
\gamma_{a}\left(t_{a}, v_{a}^{\mathrm{ue}}, u_{a}\right) & =\max _{v_{a} \geq 0} \frac{\alpha_{a}\left(\left(v_{a}^{\mathrm{ue}}\right)^{p}-\left(v_{a}\right)^{p}\right) v_{a}+\left(v_{a}-v_{a}^{\mathrm{ue}}\right) u_{a}}{\left(t_{a}^{0}+\alpha_{a}\left(v_{a}\right)^{p}\right) v_{a}} \\
& \leq \max _{v_{a} \geq 0} \frac{\alpha_{a}\left(\left(v_{a}^{\mathrm{ue}}\right)^{p}-\left(v_{a}\right)^{p}\right) v_{a}+\left(v_{a}-v_{a}^{\mathrm{ue}}\right) u_{a}}{\left(\alpha_{a}\left(v_{a}\right)^{p}\right) v_{a}}
\end{aligned}
$$

where the inequality holds for any positive value of numerator and $t_{a}^{0} \geq 0$ for all $a \in A$. This is true because, for the maximization of $\gamma_{a}\left(t_{a}, v_{a}^{\mathrm{ue}}, u_{a}\right)$ and the inequality (9), we only need to consider $v_{a}$, for which $\alpha_{a}\left(\left(v_{a}^{\mathrm{ue}}\right)^{p}-\left(v_{a}\right)^{p}\right) v_{a}+\left(v_{a}-v_{a}^{\mathrm{ue}}\right) u_{a}>0$. This means that the maximum value of $\gamma_{a}\left(t_{a}, v_{a}^{\mathrm{ue}}, u_{a}\right)$ can be obtained by taking $t_{a}^{0}=0$ for the specific class of BPR link cost functions.

Now we are at the stage to find the optimal $v_{a}^{*}$ for the maximization problem (36). Define

$$
F\left(v_{a}\right)=\frac{\alpha_{a}\left(\left(v_{a}^{\mathrm{ue}}\right)^{p}-\left(v_{a}\right)^{p}\right) v_{a}+\left(v_{a}-v_{a}^{\mathrm{ue}}\right) u_{a}}{\left(\alpha_{a}\left(v_{a}\right)^{p}\right) v_{a}}
$$

Let $\mathrm{d} F\left(v_{a}\right) / \mathrm{d} v_{a}=0$, we have

$$
\frac{\alpha_{a}\left(v_{a}^{\mathrm{ue}}\right)^{p}+\kappa_{a} \alpha_{a} p\left(v_{a}^{\mathrm{ue}}\right)^{p}}{\kappa_{a} \alpha_{a} p\left(v_{a}^{\mathrm{ue}}\right)^{p+1}}=\frac{\alpha_{a}\left(v_{a}^{*}\right)^{p}+\alpha_{a} p\left(v_{a}^{*}\right)^{p}}{\alpha_{a} p\left(v_{a}^{*}\right)^{p+1}}
$$

where $\kappa_{a}$ is defined in (33). Solving the equation yields

$$
v_{a}^{*}=\left(\frac{1+p}{p}\right)\left(\frac{\kappa_{a} p}{1+\kappa_{a} p}\right) v_{a}^{\mathrm{ue}}
$$

Then from (36), we have

$$
\gamma_{a}\left(t_{a}, v_{a}^{\mathrm{ue}}, u_{a}\right) \leq \kappa_{a}\left(\frac{v_{a}^{\mathrm{ue}}}{v_{a}^{*}}\right)^{p+1}-1=\kappa_{a}\left[\left(\frac{p}{1+p}\right)\left(\frac{1+\kappa_{a} p}{\kappa_{a} p}\right)\right]^{p+1}-1
$$

and

$$
\gamma_{2}\left(\mathcal{C}_{p}, u\right)=\max _{t_{a} \in \mathcal{C}_{p}, a \in A_{2}} \gamma_{a}\left(t_{a}, v_{a}^{\mathrm{ue}}, u_{a}\right)=\max _{a \in A_{2}} \kappa_{a}\left[\left(\frac{p}{1+p}\right)\left(\frac{1+\kappa_{a} p}{\kappa_{a} p}\right)\right]^{p+1}-1
$$

where $t_{a} \in \mathcal{C}_{p}$ is again dropped from the second "max" for the same reason for (35). 
If we further assume that the second-best pricing scheme, $u$, is chosen such that $\kappa_{a} \equiv \kappa$ for all $a \in A$, then finally we arrive at

$$
\gamma\left(\mathcal{C}_{p}, u\right)= \begin{cases}\frac{(1+\kappa p) p}{1+p}\left(\frac{(1+\kappa p)}{(1+p)}\right)^{\frac{1}{p}}-\kappa p, & \text { if } 0 \leq \kappa \leq 1 \\ \kappa\left[\left(\frac{p}{1+p}\right)\left(\frac{1+\kappa p}{\kappa p}\right)\right]^{p+1}-1, & \text { if } \kappa \geq 1\end{cases}
$$

Clearly, if $\kappa=0 \quad(u=0)$, or in the absence of toll pricing, we have

$$
\gamma\left(\mathcal{C}_{p}, u\right)_{u=0}=p(1+p)^{-\frac{p+1}{p}}
$$

as a result,

$$
\begin{aligned}
& \rho_{\mathrm{fd}}^{\mathrm{ue}}(u)_{u=0} \leq \frac{1}{1-\gamma\left(\mathcal{C}_{p}\right)}=\left(1-p(1+p)^{-\frac{p+1}{p}}\right)^{-1} \\
& \phi_{\mathrm{fd}}^{\mathrm{ue}}(u)_{u=0}=\rho_{\mathrm{fd}}^{\mathrm{ue}}(u)_{u=0}-1 \leq p(1+p)^{-\frac{p+1}{p}}\left(1-p(1+p)^{-\frac{p+1}{p}}\right)^{-1}
\end{aligned}
$$

We thus have the same result of the price of anarchy for non-atomic congestion game with polynomial link cost functions established in the literature (Roughgarden and Tardos, 2002) (here it should be noted that Roughgarden and Tardos (2002) considered general polynomials with nonnegative coefficients and degree at most $p$, rather than monomials of degree $p$ plus a constant considered above). If we further assume that $p=1$ in (41)-(43) then we have $\gamma=1 / 4, \rho_{\mathrm{fd}}^{\text {ue }}=4 / 3$ and $\phi_{\mathrm{fd}}^{\text {ue }}=1 / 3$, the same result as obtained by Roughgarden and Tardos (2002) for a class of linear cost functions. For BPR type link cost functions with $p=4$, we have $\gamma=4 / 5^{5 / 4} \approx 0.5350, \rho_{\mathrm{fd}}^{\text {ue }}=2.1505$ and $\phi_{\mathrm{fd}}^{\text {ue }}=1.1505$. In addition, $\rho_{\mathrm{fd}}^{\text {ue }} \rightarrow 1$ and $\phi_{\mathrm{fd}}^{\mathrm{ue}} \rightarrow 0$ as $p \rightarrow 0 \quad$ (without traffic congestion), but $\rho_{\mathrm{fd}}^{\text {ue }} \rightarrow \infty$ and $\phi_{\mathrm{fd}}^{\text {ue }} \rightarrow \infty$ as $p \rightarrow+\infty$ (with severe congestion).

Furthermore, if $\kappa_{a} \equiv \kappa \equiv 1$ for all $a \in A$, or in the case of a perfect marginal-cost pricing scheme: $u_{a}=v_{a}^{\mathrm{ue}} t_{a}^{\prime}\left(v_{a}^{\mathrm{ue}}\right)$ for all $a \in A$, we obtain $\gamma(\mathcal{C}, u)=0$ from (40) and thus $\phi_{\mathrm{fd}}^{\mathrm{ue}}(u)=0$. This result simply implies that "there is no efficiency loss" or "the UE flow pattern is also system-optimal" under the marginal-cost pricing scheme.

Example 1 Consider a traffic network with the BPR-type link cost function (30). Let $v^{\text {ue }}(u)$ be the UE link flow pattern on the network associated with a pricing scheme $u$. If 
we assume that $u_{a}=\kappa\left[v_{a}^{\mathrm{ue}} t_{a}^{\prime}\left(v_{a}^{\mathrm{ue}}\right)\right]=\kappa \alpha_{a} p\left(v_{a}^{\mathrm{ue}}\right)^{p}$ for each link $a \in A$, then, from (21) and (22), the relative efficiency loss in relation to the system optimum, associated with $u$, is given by

$$
\phi_{\mathrm{fd}}^{\mathrm{ue}}(u) \leq\left\{\begin{array}{l}
\frac{\gamma\left(\mathcal{C}_{p}, u\right)}{1-\gamma\left(\mathcal{C}_{p}, u\right)}, \text { if } 0 \leq \kappa \leq 1 \\
\gamma\left(\mathcal{C}_{p}, u\right), \text { if } \kappa \geq 1
\end{array}\right.
$$

where $\gamma\left(\mathcal{C}_{p}, u\right)$ is given by (40) for the class of BPR-type link cost function $\mathcal{C}_{p}$.

Figure 4 plots the relationships between parameter $\kappa$ in the interval $0 \leq \kappa \leq 3.0$ and the maximum efficiency loss $\varphi_{\mathrm{fd}}^{\text {ue }}(u)$ for $p=1$ (linear), $p=2$ (quadratic), $p=3$ (cubic) and $p=4$ (BPR functions of degree 4). In this figure, the values of efficiency loss at $u=0$ correspond to the early bounding results (43) without pricing.

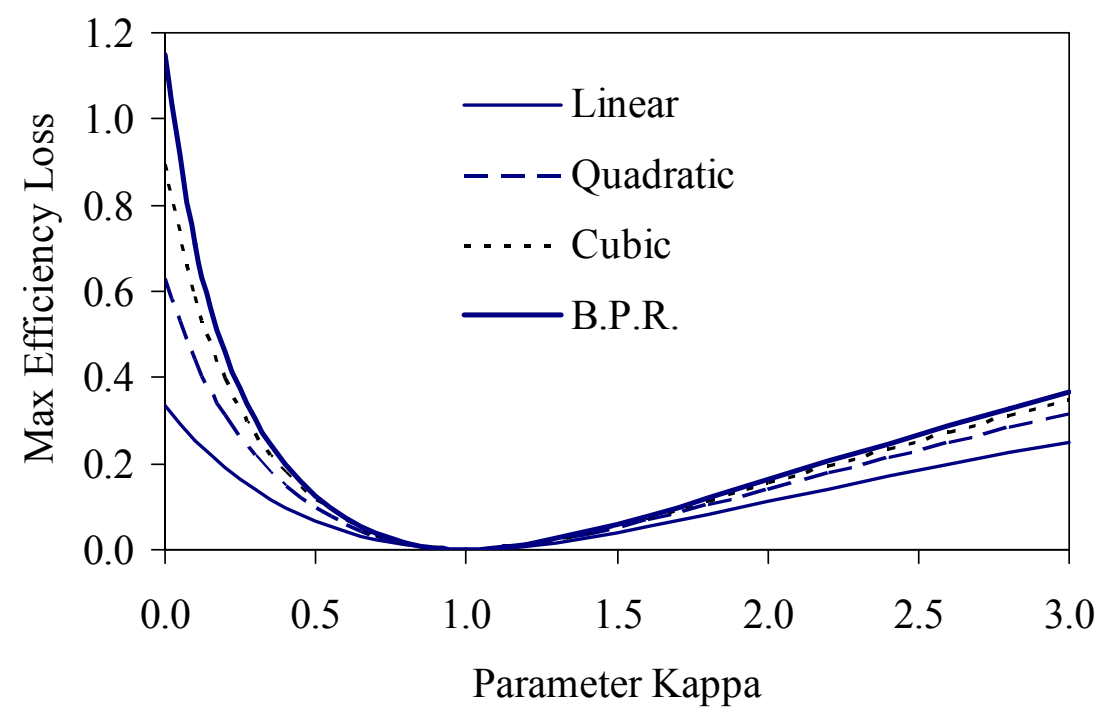

Figure 4. The maximum inefficiency loss associated with a second-best pricing scheme for BPR-type cost functions

\section{EfFiciency Gain AND Loss of PRICING With Elastic DeMAND}

\subsection{Traffic Equilibria and Inefficiency Measure}

Suppose the OD demand is a function of the equilibrium OD travel time or cost between that OD pair (for simplicity, we consider separable demand functions only), namely 
$d_{w}=D_{w}\left(\mu_{w}\right)$ where $\mu_{w} \geq 0$ is the minimum path cost between OD pair $w \in W$. We further assume that the demand function is a strictly monotone, invertible and decreasing function of travel cost. Let $\mu_{w}=D_{w}^{-1}\left(d_{w}\right)=B_{w}\left(d_{w}\right), w \in W$ denote the inverse or benefit function, which can be regarded as an amount that a user is willing to pay for his or her travel (inclusive of toll charge if any), or a benefit that he or she can obtain from this travel. In this case, the following social welfare (or net economic benefit) can serve as a meaningful system performance measure (Yang and Huang, 1998):

$$
S(v, d)=\sum_{w \in W} \int_{0}^{d_{w}} B_{w}(\omega) \mathrm{d} \omega-\sum_{a \in A} t_{a}\left(v_{a}\right) v_{a}
$$

where $B_{w}\left(d_{w}\right), w \in W$ is a decreasing function. The elastic demand UE formulation with a pricing scheme $u=\left(u_{a}: a \in A\right)^{\mathrm{T}}$, is given as

$$
\max _{(v, d) \in \Omega_{v, d}} \sum_{w \in W} \int_{0}^{d_{w}} B_{w}(\omega) \mathrm{d} \omega-\sum_{a \in A} \int_{0}^{v_{a}}\left\{t_{a}(\omega)+u_{a}\right\} \mathrm{d} \omega
$$

and the elastic demand SO formulation is given by:

$$
\max _{(v, d) \in \Omega_{v, d}} \sum_{w \in W} \int_{0}^{d_{w}} B_{w}(\omega) \mathrm{d} \omega-\sum_{a \in A} t_{a}\left(v_{a}\right) v_{a}
$$

where $\Omega_{v, d}$ is defined by:

$$
\Omega_{v, d}=\{(v, d) \mid v=\Delta f, \Lambda f=d, f \geq 0, d \geq 0\}
$$

where $\Delta$ and $\Lambda$ are the link/path and OD/path incidence matrix, respectively.

Let $\left(v^{\text {ue }}(u), d^{\text {ue }}(u)\right)$ be the solution of the UE problem (46) associated with a pricing scheme $u$ and $\left(v^{\text {so }}, d^{\text {so }}\right)$ be the solution of the SO problem (47), respectively. For the elastic-demand traffic equilibrium problem, we define the inefficiency of selfish user equilibria under a given pricing scheme $u$ as follows:

$$
\begin{aligned}
\rho_{\text {ed }}^{\text {ue }}(u) & =\frac{S\left(v^{\text {so }}, d^{\text {so }}\right)}{S\left(v^{\text {ue }}(u), d^{\text {ue }}(u)\right)}(\geq 1) \\
\phi_{\text {ed }}^{\text {ue }}(u) & =\frac{S\left(v^{\text {so }}(u), d^{\text {so }}(u)\right)-S\left(v^{\text {ue }}(u), d^{\text {ue }}(u)\right)}{S\left(v^{\text {so }}, d^{\text {so }}\right)}=1-\frac{1}{\rho_{\text {ed }}^{\text {ue }}(u)} \quad(\geq 0)
\end{aligned}
$$

where 'ed' denotes the case of elastic demand. Clearly, $\rho_{\text {ed }}^{\text {ue }}(u) \geq 1$ and hence $\phi_{\text {ed }}^{\text {ue }}(u) \geq 0$ hold since the UE does not maximize the objective function defined by (45). Again we attempt to seek an upper bound of $\rho_{\text {ed }}^{\text {ue }}(u)$ and hence the upper bound of $\phi_{\text {ed }}^{\text {ue }}(u)$ to quantify the maximum efficiency loss of a general second-best pricing scheme in comparison with the social optimum case. As a special case by letting $u=0$, our bounding result will naturally 
quantify the maximum potential of raising total social welfare or maximum efficiency gain by a marginal-cost pricing scheme.

The price of anarchy with elastic demand was examined by Chau and Sim (2003) in the absence of toll pricing. Here we first use a simple example to show that finding the bound of $\rho_{\mathrm{ed}}^{\text {ue }}$ in the elastic demand case is not as manageable as in the fixed demand case, even if all functions of the system are linear (see, Chau and Sim, 2003, with errors corrected here).

Example 2 Consider a network with one OD pair and one link, where the link travel time function is $t(v)=a v+b, a \geq 0$ and the benefit function $B(d)=m d+n, m<0$. The UE solution and the corresponding social welfare without pricing are

$$
v^{\text {ue }}=d^{\text {ue }}=\frac{n-b}{a-m}, \quad S\left(v^{\text {ue }}, d^{\text {ue }}\right)=-\frac{m(n-b)^{2}}{2(a-m)^{2}}
$$

The SO solution and the corresponding social welfare are

$$
v^{\mathrm{so}}=d^{\mathrm{so}}=\frac{n-b}{2 a-m}, \quad S\left(v^{\mathrm{so}}, d^{\mathrm{so}}\right)=\frac{(n-b)^{2}}{2(2 a-m)}
$$

Hence, we have

$$
\rho_{\mathrm{ed}}^{\mathrm{ue}}=\frac{S\left(v^{\mathrm{so}}, d^{\mathrm{so}}\right)}{S\left(v^{\mathrm{ue}}, d^{\text {ue }}\right)}=\frac{(m-a)^{2}}{m(m-2 a)}=\left(1-\left(\frac{a}{m-a}\right)^{2}\right)^{-1}
$$

Unlike the bounded for the fixed demand case ( $4 / 3$ for linear cost function), there is no deterministic upper bound independent of $m$. In the fixed demand case, the price of anarchy of the UE is not too severe since it is bounded, and thus the efficiency gain of the marginalcost pricing is mild. However, in the presence of elastic demand, as considered in this simple example of a linear setting, the price of anarchy of an elastic-demand UE can be dramatic if $m \rightarrow 0$ regardless of the value of parameter $a$ (it can be easily checked that in this case the elasticity of demand becomes ' $-\infty$ ' or the demand becomes perfectly elastic).

\subsection{Bound for Efficiency Gain and Loss}

In what follows, we attempt to derive a pseudo-approximation bound of $\rho_{\text {ed }}^{\text {ue }}$ in terms of the social welfare and user benefit at a given user optimum solution and the parameter $\gamma(\mathcal{C}, u)$ established in previous section. Then, we consider how to obtain a relaxed upper bound that depends on the class of link cost functions and the demand elasticity only to get ride of the specific demand functions. The relaxed upper bound will suffice for us to determine the welfare gap between a second-best pricing scheme and the system optimum (or the first-best pricing scheme), the gap will completely disappear as the second-best pricing scheme approaches the first-best one. We first introduce the following lemma (Chau and Sim, 2003). 
Lemma 1 If $B_{w}\left(d_{w}\right)$ is a non-increasing function of $d_{w}$ for $d_{w}>0$, then

$$
\sum_{w \in W} \int_{0}^{d_{w}} B_{w}(\omega) \mathrm{d} \omega \leq \sum_{w \in W} \int_{0}^{d_{w}^{\mathrm{ue}}} B_{w}(\omega) \mathrm{d} \omega+\sum_{w \in W} B_{w}\left(d_{w}^{\mathrm{ue}}\right)\left(d_{w}-d_{w}^{\mathrm{ue}}\right)
$$

Proof: Since $B_{w}\left(d_{w}\right)$ is non-increasing, we have

$$
\left(d_{w}-d_{w}^{\text {ue }}\right) B_{w}\left(d_{w}^{\text {ue }}\right) \geq \int_{d_{w}^{\text {ue }}}^{d_{w}} B_{w}(\omega) \mathrm{d} \omega, w \in W
$$

which can be rewritten as

$$
\int_{0}^{d_{w}} B_{w}(\omega) \mathrm{d} \omega \leq \int_{0}^{d_{w}^{\text {ue }}} B_{w}(\omega) \mathrm{d} \omega+\left(d_{w}-d_{w}^{\text {ue }}\right) B_{w}\left(d_{w}^{\text {ue }}\right), w \in W
$$

Summing up over all OD pairs $w \in W$ leads to (51).

Theorem 3 Let $v^{\text {ue }}(u)$ be an elastic-demand UE link flow pattern associated with a pricing scheme $u$, with separable cost functions drawn from a given class $\mathcal{C}$, and let $v^{\text {so }}$ be an SO link flow solution, then

$$
\begin{aligned}
& \rho_{\text {ed }}^{\text {ue }}(u) \leq 1+\left(\omega_{1}(u)-1\right) \gamma(\mathcal{C}, u), \text { if } 0 \leq u_{a} \leq v_{a}^{\text {ue }} t_{a}^{\prime}\left(v_{a}^{\text {ue }}\right), \forall a \in A \\
& \rho_{\text {ed }}^{\text {ue }}(u) \leq \frac{1+\omega_{2}(u) \gamma(\mathcal{C}, u)}{1+\gamma(\mathcal{C}, u)}, \text { if } u_{a} \geq v_{a}^{\text {ue }} t_{a}^{\prime}\left(v_{a}^{\text {ue }}\right), \forall a \in A
\end{aligned}
$$

where

$$
\begin{aligned}
& \omega_{1}(u)=\frac{U\left(d^{\mathrm{ue}}(u)\right)}{S\left(v^{\mathrm{ue}}(u), d^{\mathrm{ue}}(u)\right)} \text { for } 0 \leq u_{a} \leq v_{a}^{\mathrm{ue}} t_{a}^{\prime}\left(v_{a}^{\mathrm{ue}}\right), \forall a \in A \\
& \omega_{2}(u)=\frac{U\left(d^{\mathrm{so}}\right)}{S\left(v^{\mathrm{ue}}(u), d^{\mathrm{ue}}(u)\right)} \text { for } u_{a} \geq v_{a}^{\mathrm{ue}} t_{a}^{\prime}\left(v_{a}^{\mathrm{ue}}\right), \forall a \in A
\end{aligned}
$$

and $S\left(v^{\text {ue }}(u), d^{\text {ue }}(u)\right)$ is the social welfare defined in (45) at UE associated with pricing scheme $u ; U\left(d^{\mathrm{ue}}(u)\right)$ and $U\left(d^{\mathrm{so}}\right)$ are the user benefits at $U E$ with pricing scheme $u$ and at $S O$, respectively:

$$
U\left(d^{\text {ue }}(u)\right)=\sum_{w \in W} \int_{0}^{d_{w}^{\text {ue }}(u)} B_{w}(\omega) \mathrm{d} \omega ; \quad U\left(d^{\mathrm{so}}\right)=\sum_{w \in W} \int_{0}^{d_{w}^{\mathrm{so}}} B_{w}(\omega) \mathrm{d} \omega
$$

Proof: Since $\left(v^{\text {ue }}, d^{\text {ue }}\right)$ is an equilibrium solution, from VI formulation of the elasticdemand traffic equilibrium problem with a pricing scheme $u$, we have: 


$$
\sum_{a \in A}\left(t_{a}\left(v_{a}^{\mathrm{ue}}\right)+u_{a}\right)\left(v_{a}-v_{a}^{\mathrm{ue}}\right)-\sum_{w \in W} B_{w}\left(d_{w}^{\mathrm{ue}}\right)\left(d_{w}-d_{w}^{\mathrm{ue}}\right) \geq 0, \forall(v, d) \in \Omega_{v, d}
$$

Substituting (51) into (59) yields

$$
\sum_{a \in A}\left(t_{a}\left(v_{a}^{\mathrm{ue}}\right)+u_{a}\right)\left(v_{a}-v_{a}^{\mathrm{ue}}\right)+\sum_{w \in W} \int_{0}^{d_{w}^{\mathrm{ue}}} B_{w}(\omega) \mathrm{d} \omega-\sum_{w \in W} \int_{0}^{d_{w}} B_{w}(\omega) \mathrm{d} \omega \geq 0
$$

It can be rewritten as

$$
\begin{aligned}
\sum_{w \in W} \int_{0}^{d_{w}^{\mathrm{ue}}} B_{w}(\omega) \mathrm{d} \omega-\sum_{a \in A} t_{a}\left(v_{a}^{\mathrm{ue}}\right) & v_{a}^{\mathrm{ue}}-\left\{\sum_{w \in W} \int_{0}^{d_{w}} B_{w}(\omega) \mathrm{d} \omega-\sum_{a \in A} t_{a}\left(v_{a}\right) v_{a}\right\} \\
+ & \sum_{a \in A}\left(t_{a}\left(v_{a}^{\mathrm{ue}}\right)-t_{a}\left(v_{a}\right)\right) v_{a}+\sum_{a \in A}\left(v_{a}-v_{a}^{\mathrm{ue}}\right) u_{a} \geq 0
\end{aligned}
$$

If $0 \leq u_{a} \leq v_{a}^{\mathrm{ue}} t_{a}^{\prime}\left(v_{a}^{\mathrm{ue}}\right), \forall a \in A$, from definitions (10) and (16) or using eqn. (19) directly, we have:

$$
S\left(v^{\text {ue }}(u), d^{\text {ue }}(u)\right)-S(v, d)+\gamma(\mathcal{C}, u) T\left(v^{\text {ue }}(u)\right) \geq 0
$$

Using definition (49) and let $(v, d)=\left(v^{\text {so }}, d^{\text {so }}\right)$ in (60), we obtain

$$
\rho_{\text {ed }}^{\text {ue }} \leq 1+\gamma(\mathcal{C}, u) \frac{T\left(v^{\text {ue }}(u)\right)}{S\left(v^{\text {ue }}(u), d^{\text {ue }}(u)\right)}
$$

Using $T\left(v^{\text {ue }}(u)\right)=-S\left(v^{\text {ue }}(u), d^{\text {ue }}(u)\right)+U\left(d^{\text {ue }}(u)\right)$, we thus obtain (54) with $\omega_{1}(u)$ given by (56).

If $u_{a} \geq v_{a}^{\mathrm{ue}} t_{a}^{\prime}\left(v_{a}^{\mathrm{ue}}\right), \forall a \in A$, using definition (11) and (16), we have

$$
S\left(v^{\text {ue }}(u), d^{\text {ue }}(u)\right)-S(v, d)+\gamma(\mathcal{C}, u) T(v) \geq 0
$$

Let $(v, d)=\left(v^{\mathrm{so}}, d^{\mathrm{so}}\right)$ in $(61)$, we have

$$
\begin{aligned}
\rho_{\text {ed }}^{\mathrm{ue}}(u) & \leq 1+\gamma(\mathcal{C}, u) \frac{T\left(v^{\mathrm{so}}\right)}{S\left(v^{\mathrm{ue}}(u), d^{\mathrm{ue}}(u)\right)}=1+\gamma(\mathcal{C}, u) \frac{U\left(d^{\mathrm{so}}\right)-S\left(v^{\mathrm{so}}, d^{\mathrm{so}}\right)}{S\left(v^{\mathrm{ue}}(u), d^{\mathrm{ue}}(u)\right)} \\
& =1+\gamma(\mathcal{C}, u)\left(-\rho_{\mathrm{ec}}^{\mathrm{ue}}(u)+\frac{U\left(d^{\mathrm{so}}\right)}{S\left(v^{\mathrm{ue}}(u), d^{\mathrm{ue}}(u)\right)}\right) \\
& =1+\gamma(\mathcal{C}, u)\left(-\rho_{\mathrm{ec}}^{\mathrm{ue}}(u)+\omega_{2}(u)\right)
\end{aligned}
$$

This yields the second case result given in (55) with $\omega_{2}(u)$ defined in (57).

Next, we eliminate the uniformity magnitude assumption of link toll charges $u$ to include both possibilities of $0 \leq u_{a} \leq v_{a}^{\text {ue }} t_{a}^{\prime}\left(v_{a}^{\text {ue }}\right)$ and $u_{a} \geq v_{a}^{\text {ue }} t_{a}^{\prime}\left(v_{a}^{\text {ue }}\right)$ in a single bound. Using the same definitions of $A_{1}, A_{2}, \gamma_{1}(\mathcal{C}, u)$ and $\gamma_{2}(\mathcal{C}, u)$, as in the fixed demand case, we now have the following Theorem. 
Theorem 4 Let $v^{\mathrm{ue}}(u)$ be an elastic-demand UE link flow pattern associated with a pricing scheme $u$, with separable cost functions drawn from a given class $\mathcal{C}$, and let $v^{\text {so }}$ be an SO link flow solution, then

$$
\rho_{\text {ed }}^{\text {ue }}(u) \leq \frac{1-\gamma_{1}(\mathcal{C}, u)}{1+\gamma_{2}(\mathcal{C}, u)}+\frac{\gamma_{1}(\mathcal{C}, u) \omega_{1}(u)+\gamma_{2}(\mathcal{C}, u) \omega_{2}(u)}{1+\gamma_{2}(\mathcal{C}, u)}
$$

where $\omega_{1}(u)$ and $\omega_{2}(u)$ are defined by (56) and (57), respectively.

Proof: Like the proof of Theorem 3 and using the relation (27), the counterpart of eqn. (60) or (61) becomes

$$
S\left(v^{\text {ue }}(u), d^{\text {ue }}(u)\right)-S(v, d)+\gamma_{1}(\mathcal{C}, u) T\left(v^{\text {ue }}(u)\right)+\gamma_{2}(\mathcal{C}, u) T(v) \geq 0
$$

Substituting $\quad T\left(v^{\text {ue }}(u)\right)=-S\left(v^{\text {ue }}(u), d^{\text {ue }}(u)\right)+U\left(d^{\text {ue }}(u)\right) \quad$ and $\quad T(v)=-S(v, d)+U(d)$ into (63) yields

$$
\begin{aligned}
\left(1-\gamma_{1}(\mathcal{C}, u)\right) S\left(v^{\text {ue }}(u), d^{\text {ue }}(u)\right)-\left(1+\gamma_{2}(\mathcal{C}, u)\right) S(v, d) \\
+\left(\gamma_{1}(\mathcal{C}, u) U\left(d^{\text {ue }}(u)\right)+\gamma_{2}(\mathcal{C}, u) U(d)\right) \geq 0
\end{aligned}
$$

Using definition (49) and let $(v, d)=\left(v^{\text {so }}, d^{\text {so }}\right)$ in (64), we have

$$
\rho_{\text {ed }}^{\text {ue }}(u) \leq \frac{1-\gamma_{1}(\mathcal{C}, u)}{1+\gamma_{2}(\mathcal{C}, u)}+\frac{\gamma_{1}(\mathcal{C}, u) \frac{U\left(d^{\text {ue }}(u)\right)}{S\left(v^{\text {ue }}(u), d^{\text {ue }}(u)\right)}+\gamma_{2}(\mathcal{C}, u) \frac{U\left(d^{\text {so }}\right)}{S\left(v^{\text {ue }}(u), d^{\text {ue }}(u)\right)}}{1+\gamma_{2}(\mathcal{C}, u)}
$$

By the definitions of $\omega_{1}(u)$ and $\omega_{2}(u)$, we obtain (62).

Theorem 4 is particularly useful for establishing the inefficiency bound or efficiency loss for practical second-best pricing schemes with elastic demands. Once $\rho_{\text {ed }}^{\text {ue }}(u)$ is determined, we can calculate the efficiency loss, $\phi_{\text {ed }}^{\text {ue }}(u)$, of the second-best pricing scheme straightforwardly using eqn. (50).

\subsection{Selection of Practical Bounding Parameter Values}

For calculation of the inefficiency bounds established above, we have to ascertain the values of parameter $\omega_{1}(u)$ and $\omega_{2}(u)$ defined in (56) and (57). If the demand function is known, it is straightforward to calculate $\omega_{1}(u)$ and $\omega_{2}(u)$, based on the known demand function and the relevant $\mathrm{SO}$ and UE solutions with pricing. Here is a simple example.

Example 3 Consider the following negative exponential demand function: 


$$
d=d_{0} \exp \left[-\alpha\left(\frac{\mu}{\mu_{0}}-1\right)\right]
$$

where $\alpha>0$ is a cost sensitivity parameter and $\mu_{0}$ is the minimum free-flow travel time and $d_{0}$ is the maximum demand when $\mu=\mu_{0}$.

The inverse demand function is given by

$$
\mu=\mu_{0}\left(1-\frac{1}{\alpha} \ln \left(\frac{d}{d_{0}}\right)\right)
$$

The user benefit, $U$, is given by

$$
U=\int_{0}^{d} \mu_{0}\left(1-\frac{1}{\alpha} \ln \left(\frac{x}{d_{0}}\right)\right) \mathrm{d} x=\mu_{0} d\left[1-\frac{1}{\alpha}\left(\ln \left(\frac{d}{d_{0}}\right)-1\right)\right]
$$

and, in the absence of pricing, the social welfare, $S$, is given by

$$
S=U-\mu d=\mu_{0} d\left[1-\frac{1}{\alpha}\left(\ln \left(\frac{d}{d_{0}}\right)-1\right)\right]-\mu_{0} d\left(1-\frac{1}{\alpha} \ln \left(\frac{d}{d_{0}}\right)\right)=\frac{1}{\alpha} \mu_{0} d
$$

Let $\omega=U / S$ be the ratio of the user benefit to social welfare for any $(\mu, d)$, then

$$
\omega=\frac{U}{S}=\frac{\mu_{0} d\left[1-\frac{1}{\alpha}\left(\ln \left(\frac{d}{d_{0}}\right)-1\right)\right]}{\frac{1}{\alpha} \mu_{0} d}=1+\alpha-\ln \left(\frac{d}{d_{0}}\right)
$$

In practice, the demand function is usually unknown. In this case, if parameters, $\omega_{1}(u)$ and $\omega_{2}(u)$, are bounded, then their approximate values should suffice to serve our purpose of bounding the inefficiency. This is due to the fact that in eqns. (54) and (55)

$$
1+\left(\omega_{1}(u)-1\right) \gamma(\mathcal{C}, u) \rightarrow 1.0 ; \quad \frac{1+\omega_{2}(u) \gamma(\mathcal{C}, u)}{1+\gamma(\mathcal{C}, u)} \rightarrow 1.0
$$

as $\gamma(\mathcal{C}, u) \rightarrow 0$ for any limited values of $\omega_{1}(u)$ and $\omega_{2}(u)$. Moreover, in eqn. (62)

$$
\frac{1-\gamma_{1}(\mathcal{C}, u)}{1+\gamma_{2}(\mathcal{C}, u)}+\frac{\gamma_{1}(\mathcal{C}, u) \omega_{1}(u)+\gamma_{2}(\mathcal{C}, u) \omega_{2}(u)}{1+\gamma_{2}(\mathcal{C}, u)} \rightarrow 1.0
$$

as $\gamma_{1}(\mathcal{C}, u) \rightarrow 0$ and $\gamma_{2}(\mathcal{C}, u) \rightarrow 0$ for any limited values of $\omega_{1}(u)$ and $\omega_{2}(u)$. To put it differently, with approximate values of $\omega_{1}(u)$ and $\omega_{2}(u)$, the convergence of a sequential experimental pricing scheme or the nearness of a given current second-best pricing scheme to the first-best one in its neighborhood, can still be well understood, without requiring the specific demand functions. The following lemma is useful for gauging a practical value of $\omega$. 
Lemma 2 If the demand function $d=D(\mu)$, where $\mu \geq 0$ is the generalized travel cost, inclusive of toll charge, if any, is monotonically decreasing and convex, then

$$
1 \leq \omega=\frac{U}{S} \leq 1-2 E_{d}^{\mu}
$$

where $U$ and $S$ denotes the user benefit and social welfare for any realized $(\mu, d)$ and $E_{d}^{\mu}$ is the price elasticity of demand at $(d, \mu)$ defined by

$$
E_{d}^{\mu}=\frac{\mathrm{d} D(\mu)}{\mathrm{d} \mu} \frac{\mu}{d}=D^{\prime}(\mu) \frac{\mu}{d} \quad(\leq 0)
$$

Proof: Consider Figure 5, where $T=t d$ denotes the total travel time cost with $t$ being the travel time, $R=u d$ denotes the toll revenue with $u$ being the toll charge, and $C S$ denotes the consumer surplus (or net user benefit) given by the area under the demand curve and above the line segment $\overline{\mathrm{ab}}$.

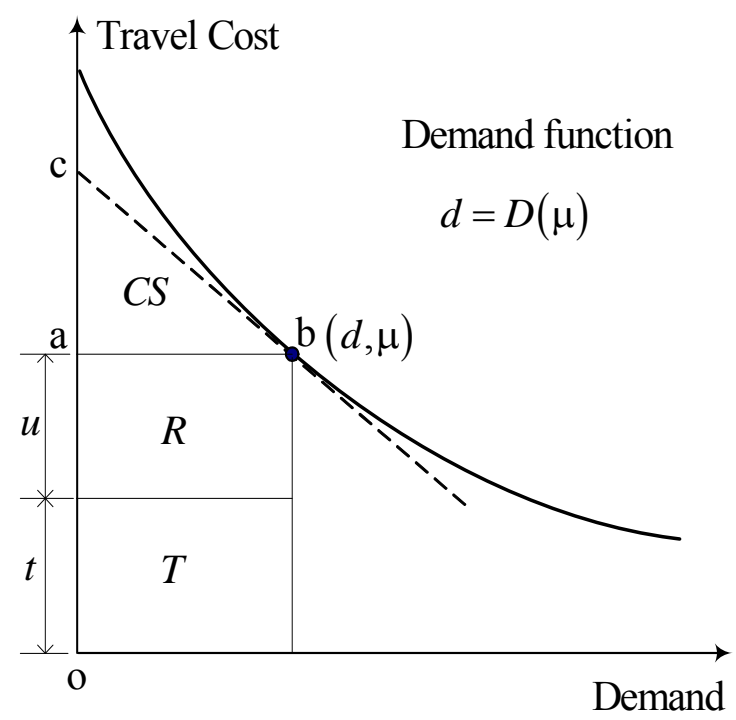

Figure 5. Geometric illustration of the proof of Lemma 2

By definition

$$
\omega=\frac{U}{S}=\frac{U}{U-T}=\frac{C S+R+T}{C S+R}=1+\frac{T}{C S+R}
$$

thus,

$$
1 \leq \omega \leq 1+\frac{T}{C S}
$$

since $R, T, C S \geq 0$. Because the demand function, $d=D(\mu)$, is monotonically decreasing 
and convex, clearly we have $C S \geq \Delta_{\mathrm{abc}}$, where $\Delta_{\mathrm{abc}}$ is the area of the triangle $\overline{\mathrm{abc}}$ with $\overline{\mathrm{bc}}$ being the tangent line of the demand curve at point $(d, \mu)$. One can easily find that

$$
\Delta_{a b c}=-\frac{1}{2} \frac{d^{2}}{D^{\prime}(\mu)} \quad \text { where } D^{\prime}(\mu)<0
$$

Hence, from definition (68) and in view of $\mu d=T+R \geq T$ with $u \geq 0$, we have

$$
1<\omega \leq 1+\frac{T}{C S} \leq 1+T\left(-\frac{1}{2} \frac{d^{2}}{D^{\prime}(\mu)}\right)^{-1}=1-2\left(\frac{T}{T+R}\right) E_{d}^{\mu} \leq 1-2 E_{d}^{\mu}
$$

The proof is completed.

Lemma 2 shows that $\omega=U / S \geq 1$ is upper-bounded by $\left(1-2 E_{d}^{\mu}\right)$ at any realized $(\mu, d)$ for any monotonically decreasing and convex demand function and any pricing scheme $u$. As we have seen from the proof, the upper bound is exactly matched for linear demand functions without pricing.

Example 4 Consider again Example 3 with negative exponential demand function (65). Applying the formula of the price elasticity of demand yields

$$
E_{d}^{\mu}=\frac{\mathrm{d} D(\mu)}{\mathrm{d} \mu} \frac{\mu}{d}=d\left(-\frac{\alpha}{\mu_{0}}\right) \frac{\mu}{d}=-\alpha \frac{\mu}{\mu_{0}}=\ln \left(\frac{d}{d_{0}}\right)-\alpha
$$

From eqn. (66), we have $\omega=1-E_{d}^{\mu}$, which meets $1 \leq \omega \leq 1-2 E_{d}^{\mu}$.

From Lemma 2 and eqn. (56), we readily have

$$
1 \leq \omega_{1}(u) \leq 1-2\left(E_{d}^{\mu}\right)_{\text {ue }}
$$

where $\left(E_{d}^{\mu}\right)_{\text {ue }}$ is the price elasticity evaluated at equilibrium demand, $d^{\text {ue }}(u)$, associated with pricing scheme $u$.

As to $\omega_{2}(u)$ defined by eqn. (57), its bound in terms of price elasticity of demand is somewhat complicated, because the user benefit, $U\left(d^{\text {so }}\right)$, and the social welfare, $S\left(v^{\text {ue }}(u), d^{\text {ue }}(u)\right)$, are evaluated at two different points, SO and UE, respectively. We have to examine the following two cases shown in Figure 6, separately. 


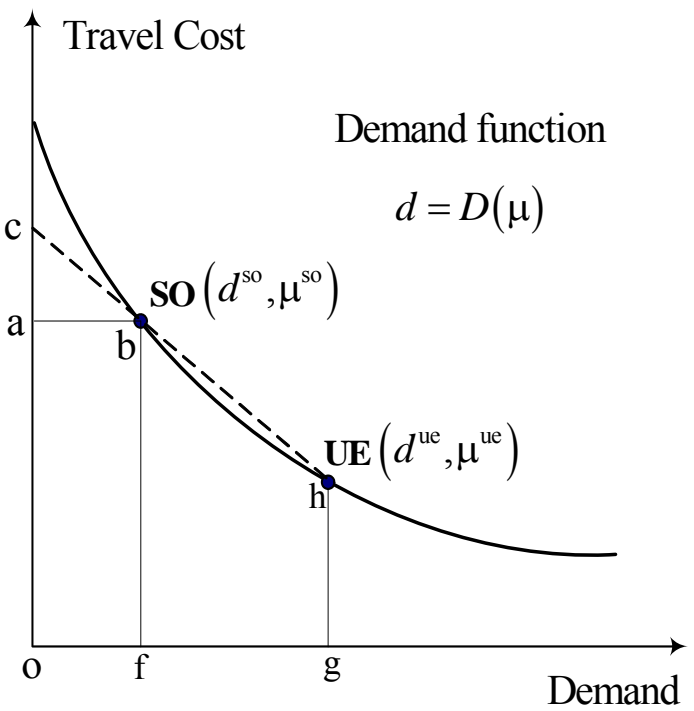

(a) UE on the right-hand side of SO

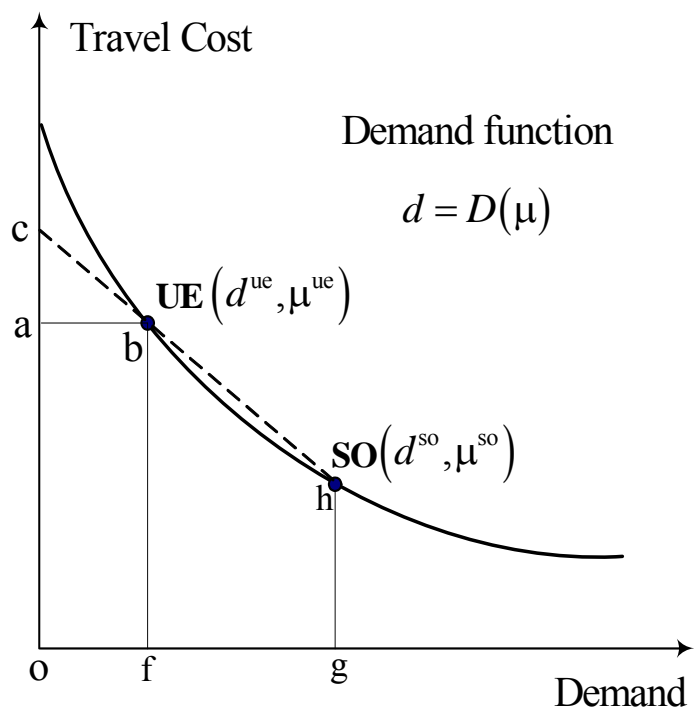

(b) UE on the left-hand side of SO

Figure 6. Geometric illustration of determination of parameter bound

Case 1: UE located on the right-hand side of SO. This case is shown in Figure 6 (a) where $\mu^{\text {so }} \geq \mu^{\text {ue }}$ and $d^{\text {so }} \leq d^{\text {ue }}$, and thus, $U\left(d^{\text {so }}\right) \leq U\left(d^{\text {ue }}(u)\right)$. From definition (57), Lemma 2 and, $S\left(v^{\text {ue }}(u), d^{\text {ue }}(u)\right) \leq S\left(v^{\text {so }}, d^{\text {so }}\right)$, we readily have

$$
1 \leq \frac{U\left(d^{\mathrm{so}}\right)}{S\left(v^{\mathrm{so}}, d^{\mathrm{so}}\right)} \leq \omega_{2}(u)=\frac{U\left(d^{\mathrm{so}}\right)}{S\left(v^{\mathrm{ue}}(u), d^{\mathrm{ue}}(u)\right)} \leq \frac{U\left(d^{\mathrm{ue}}(u)\right)}{S\left(v^{\mathrm{ue}}(u), d^{\mathrm{ue}}(u)\right)} \leq 1-2\left(E_{d}^{\mu}\right)_{\mathrm{ue}}
$$

where $\left(E_{d}^{\mu}\right)_{\text {ue }}$ is again the price elasticity of demand evaluated at UE under pricing scheme $u$. We thus have the same bounds of $\omega_{2}(u)$ as those of $\omega_{1}(u)$ in (69).

Case 2: UE located on the left-hand side of SO. This case is shown in Figure 6 (b), where $\mu^{\text {so }} \leq \mu^{\text {ue }}$ and $d^{\text {so }} \geq d^{\text {ue }}$. We thus obtain immediately that

$$
\begin{aligned}
1 \leq \omega_{2}(u)=\frac{U\left(d^{\mathrm{so}}\right)}{S\left(v^{\mathrm{ue}}(u), d^{\mathrm{ue}}(u)\right)} & \leq \frac{U\left(d^{\mathrm{ue}}\right)}{S\left(v^{\mathrm{ue}}(u), d^{\mathrm{ue}}(u)\right)}+\frac{\Delta_{\mathrm{bfgh}}}{S\left(v^{\mathrm{ue}}(u), d^{\mathrm{ue}}(u)\right)} \\
& \leq \frac{U\left(d^{\mathrm{ue}}\right)}{S\left(v^{\mathrm{ue}}(u), d^{\mathrm{ue}}(u)\right)}+\frac{\Delta_{\mathrm{bfgh}}}{\Delta_{\mathrm{abc}}}
\end{aligned}
$$

where $\Delta_{\text {bfgh }}$ and $\Delta_{\mathrm{abc}}$ denote the areas of trapezoid $\overline{\mathrm{bfgh}}$ and triangle $\overline{\mathrm{abc}}$, respectively. Moreover, from $\overline{\mathrm{ac}}=\left(\mu^{\mathrm{ue}}-\mu^{\mathrm{so}}\right) d^{\mathrm{ue}} /\left(d^{\mathrm{so}}-d^{\mathrm{ue}}\right)$, we have 


$$
\begin{aligned}
& \frac{\Delta_{\text {bfgh }}}{\Delta_{\text {abc }}}=\frac{\frac{1}{2}\left(\mu^{\text {so }}+\mu^{\text {ue }}\right)\left(d^{\text {so }}-d^{\text {ue }}\right)}{\frac{1}{2} d^{\text {ue }} \frac{d^{\text {ue }}}{\left(d^{\text {so }}-d^{\text {ue }}\right)}\left(\mu^{\text {ue }}-\mu^{\text {so }}\right)} \\
& =\left(1-\left(\frac{\mu^{\text {so }}}{\mu^{\text {ue }}}\right)^{2}\right)\left(\frac{d^{\text {so }}-d^{\text {ue }}}{\mu^{\text {so }}-\mu^{\text {ue }}} \cdot \frac{\mu^{\text {ue }}}{d^{\text {ue }}}\right)^{2} \\
& =\left(1-\left(\frac{\mu^{\mathrm{so}}}{\mu^{\mathrm{ue}}}\right)^{2}\right)\left(\left(\tilde{E}_{d}^{\mu}\right)_{\text {ue }}^{\text {so }}\right)^{2} \\
& \leq\left(\left(\tilde{E}_{d}^{\mu}\right)_{\text {ue }}^{\text {so }}\right)^{2}
\end{aligned}
$$

where

$$
\left(\tilde{E}_{d}^{\mu}\right)_{\text {ue }}^{\text {so }}=\frac{d^{\text {so }}-d^{\text {ue }}}{\mu^{\text {so }}-\mu^{\text {ue }}} \cdot \frac{\mu^{\text {ue }}}{d^{\text {ue }}}
$$

is the price elasticity of demand in terms of the shrinkage ratio defined by the two points UE and SO. It is evident that $\left(\tilde{E}_{d}^{\mu}\right)_{\mathrm{ue}}^{\mathrm{so}} \approx\left(E_{d}^{\mu}\right)_{\mathrm{ue}} \approx\left(E_{d}^{\mu}\right)_{\mathrm{so}}$ as the current pricing scheme approaches the first-best one. Substituting eqn. (72) and (70) into (71) yields

$$
1 \leq \omega_{2}(u)=\frac{U\left(d^{\text {so }}\right)}{S\left(v^{\text {ue }}(u), d^{\text {ue }}(u)\right)} \leq 1-2\left(E_{d}^{\mu}\right)_{\text {ue }}+\left(\left(\tilde{E}_{d}^{\mu}\right)_{\text {ue }}^{\text {so }}\right)^{2}
$$

Summarizing the above discussion on both cases, we can see that $\omega_{2}(u)$ is upper bounded by the term of the right-hand side in (74) in terms of proper price elasticity of demand associated with UE and SO points.

For the general network case, because the user benefit and social welfare are additive with respect to OD pairs, we have immediately that

$$
\begin{aligned}
\omega_{1}=\frac{\sum_{w \in W} U_{w}^{\text {ue }}}{\sum_{w \in W} S_{w}^{\text {ue }}} \leq \frac{\sum_{w \in W}\left(1-2\left(E_{d_{w}}^{\mu_{w}}\right)_{\text {ue }}\right) S_{w}^{\text {ue }}}{\sum_{w \in W} S_{w}^{\text {ue }}} & \leq \frac{\sum_{w \in W} S_{w}^{\text {ue }}}{\sum_{w \in W} S_{w}^{\text {ue }}} \max _{w \in W}\left(1-2\left(E_{d_{w}}^{\mu_{w}}\right)_{\text {ue }}\right) \\
& =\max _{w \in W}\left(1-2\left(E_{d_{w}}^{\mu_{w}}\right)_{\text {ue }}\right)
\end{aligned}
$$

where $U_{w}^{\text {ue }}, S_{w}^{\text {ue }}$ and $\left(E_{d_{w}}^{\mu_{w}}\right)_{\text {ue }}$ denotes, respectively, the user benefit, social welfare and price elasticity of demand associated with OD pair $w \in W$ at UE. Similarly,

$$
\omega_{2}=\frac{\sum_{w \in W} U_{w}^{\text {so }}}{\sum_{w \in W} S_{w}^{\text {ue }}} \leq \max _{w \in W}\left(1-2\left(E_{d}^{\mu}\right)_{\text {ue }}+\left(\left(\tilde{E}_{d_{w}}^{\mu_{w}}\right)_{\text {ue }}^{\text {so }}\right)^{2}\right)
$$

where $U_{w}^{\text {so }}$ and $\left(\tilde{E}_{d_{w}}^{\mu_{w}}\right)_{\text {ue }}^{\text {so }}$ denotes, respectively, the user benefit at SO and the price elasticity 
of demand in terms of shrinkage ratio between UE and SO, associated with OD pair $w \in W$.

If the demand within the considered range is inelastic or if $-1 \leq E_{d}^{\mu} \leq 0$ and $-1 \leq\left(\tilde{E}_{d_{w}}^{\mu_{w}}\right)_{\text {ue }}^{\text {so }} \leq 0$, then we have $1 \leq \omega_{1} \leq 3$ and $1 \leq \omega_{2} \leq 4$. The practical value of elasticity of travel demand is approximately in the range between -0.3 and -0.5 (see, Oum et al. (1992) and Goodwin (1992), for surveys of travel demand elasticity), and one may thus choose a practical value of $\omega_{1}$ within the range of $1.0 \leq \omega_{1} \leq 2.0$ and $\omega_{2}$ in the range of $1.0 \leq \omega_{2} \leq 2.25$. Once an appropriate value of $\omega$ is selected based on an approximate value of the price elasticity of demand (or qualitative property of the demand function, the bounds can be calculated in a simple manner as the fixed demand case, without requiring the specific demand function form. Note that knowing the approximate elasticity of demand is far less than knowing the demand function itself. Also, from a practical viewpoint, demand elasticity can be estimated based on the observed change in demand in response to a change in price without necessarily knowing its explicit function form.

We conclude this section by providing the following remark on the two types of inefficiency bounds established thus far for networks with fixed and elastic demands.

Remark 3 The inefficiency bounds for the elastic demand case established in this section cannot be generalized to those for the fixed demand case established in Section (2). This is due to the different measures of system inefficiency/efficiency in the two cases. The ratio of $\rho_{\mathrm{ed}}^{\mathrm{ue}}=S^{\text {so }} / S^{\text {ue }}$ in (49) for the elastic demand case is valid only for $S>0$, here $S^{\text {so }}$ and $S^{\text {ue }}$ denote social welfare $S$ at SO and UE. For the fixed demand case, one may regard $S=-T$ (negative total system travel time), in this case applying definition (49) gives rise to $S^{\text {so }} / S^{\text {ue }}=-T^{\text {so }} /-T^{\text {ue }}=T^{\text {so }} / T^{\text {ue }} \leq 1.0$, a meaningless ratio contrary with the inefficiency measure (6) for the fixed demand case. Indeed, for fixed demand case we have $E_{d}^{\mu}=0.0$ and thus $\omega_{1}=\omega_{2}=1.0$ in (75) and (76), then from (54) and (55) we always have $\rho_{\mathrm{ed}}^{\text {ue }}=S^{\mathrm{so}} / S^{\text {ue }} \leq 1.0$, which again failed to measure the system inefficiency for the fixed demand case.

\section{Alternative Approach to Efficiency Bounding}

In the previous analysis, we defined $\gamma_{a}\left(t_{a}, v_{a}^{\text {ue }}, u_{a}\right)$ differently for the two cases of $0 \leq u_{a} \leq v_{a}^{\mathrm{ue}} t_{a}^{\prime}\left(v_{a}^{\mathrm{ue}}\right)$ and $u_{a} \geq v_{a}^{\mathrm{ue}} t_{a}^{\prime}\left(v_{a}^{\mathrm{ue}}\right)$, and then reached a bound of the efficiency loss by 
introducing the following relaxation $\left(A_{1}\right.$ and $A_{2}$ are relaxed to the whole set $A$ in the summation of link travel time) in (27):

$$
\sum_{a \in A_{1}} t_{a}\left(v_{a}^{\mathrm{ue}}\right) v_{a}^{\mathrm{ue}} \leq T\left(v^{\mathrm{ue}}\right)=\sum_{a \in A} t_{a}\left(v_{a}^{\mathrm{ue}}\right) v_{a}^{\mathrm{ue}} ; \quad \sum_{a \in A_{2}} t_{a}\left(v_{a}\right) v_{a} \leq T(v)=\sum_{a \in A} t_{a}\left(v_{a}\right) v_{a}
$$

This relaxation may, however, potentially make the inefficiency bound loose. To avoid this relaxation, we now propose an alternative approach.

Redefine a unified $\gamma_{a}\left(t_{a}, v_{a}^{\text {ue }}, u_{a}\right)$ for all links, irrespective of their levels of toll charge:

$$
\gamma_{a}\left(t_{a}, v_{a}^{\mathrm{ue}}, u_{a}\right)=\max _{v_{a} \geq 0} \frac{\left(t_{a}\left(v_{a}^{\mathrm{ue}}\right)-t_{a}\left(v_{a}\right)\right) v_{a}+\left(v_{a}-v_{a}^{\mathrm{ue}}\right) u_{a}}{t_{a}\left(v_{a}^{\mathrm{ue}}\right) v_{a}^{\mathrm{ue}}+t_{a}\left(v_{a}\right) v_{a}}
$$

and

$$
\gamma(\mathcal{C}, u)=\max _{t_{a} \in \mathcal{C}, a \in A} \gamma_{a}\left(t_{a}, v_{a}^{\mathrm{ue}}, u_{a}\right)
$$

Then we do not need to do further relaxation as previously in (27). Instead, in the same spirit as before, we have the following straightforward results by reference to Theorem 2 and Theorem 4.

i) The bound for traffic equilibria with fixed demand:

$$
\rho_{\mathrm{fd}}^{\mathrm{ue}}(u) \leq \frac{1+\gamma(\mathcal{C}, u)}{1-\gamma(\mathcal{C}, u)}
$$

ii) The bound for traffic equilibria with elastic demand

$$
\rho_{\text {ed }}^{\text {ue }}(u) \leq \frac{1-\gamma(\mathcal{C}, u)}{1+\gamma(\mathcal{C}, u)}+\frac{\gamma(\mathcal{C}, u)\left(\omega_{1}(u)+\omega_{2}(u)\right)}{1+\gamma(\mathcal{C}, u)}
$$

Here, to find the value of $\gamma(\mathcal{C}, u)$, the key problem is still to determine the maximum of the following function for given $u_{a}$ :

$$
F\left(v_{a}\right)=\frac{\left(t_{a}\left(v_{a}^{\mathrm{ue}}\right)-t_{a}\left(v_{a}\right)\right) v_{a}+\left(v_{a}-v_{a}^{\mathrm{ue}}\right) u_{a}}{t_{a}\left(v_{a}^{\mathrm{ue}}\right) v_{a}^{\mathrm{ue}}+t_{a}\left(v_{a}\right) v_{a}}
$$

Any analytical solution for $\max F\left(v_{a}\right)$ is difficult, if not impossible, even for the specific polynomial cost functions examined in the previous section.

\section{Application to Practical Pricing Schemes}

Yang et al. (2004) developed an efficient and practical trial-and-error implementation scheme 
of the first-best marginal-cost pricing problem when the demand function is unknown, as is generally the case in practice. The central idea of the scheme is to adjust the link toll charges in a sequential manner, by observing and comparing the users' responses (observed aggregate link flows) and the intended or targeted flow levels adopted for determination of toll charges. An exact first-best or marginal-cost pricing scheme is ensured if the observed and targeted link flows match each other. Specifically, the sequential experimental pricing scheme and its convergence property can be described below (Yang et al., 2004).

Sequential Experimental Pricing Scheme: At a general trial $k(k=0,1,2, \cdots)$, with the link toll charge calculated by

$$
u_{a}^{(k)}=v_{a}^{(k)} t_{a}^{\prime}\left(v_{a}^{(k)}\right), a \in A
$$

where $v_{a}^{(k)}, a \in A$ is the intended or targeted link flow for the current link toll charge. Let $\left\{\hat{v}_{a}^{(k)}: a \in A\right\}$ be the revealed link flow pattern observed after imposing the current toll charge $\left\{u_{a}^{(k)}: a \in A\right\}$, then the targeted link flow for determination of link tolls for subsequent trial experiment is given by

$$
v_{a}^{(k+1)}=v_{a}^{(k)}+\alpha^{(k)}\left(\hat{v}_{a}^{(k)}-v_{a}^{(k)}\right), a \in A
$$

Theorem 5 If the predetermined sequence $\left\{\alpha^{(k)}\right\}$ in (83) satisfies the conditions:

$$
0<\alpha^{(k)} \leq 1 ; \sum_{k=1}^{\infty} \alpha^{(k)}=+\infty ; \sum_{k=1}^{\infty}\left(\alpha^{(k)}\right)^{2}<+\infty
$$

then the sequential experimental pricing scheme generates the link flows and link tolls with the following convergence property:

$$
v_{a}^{(k)} \rightarrow v_{a}^{\mathrm{so}} ; \quad u_{a}^{k} \rightarrow u_{a}^{\mathrm{mc}} ;\left|\hat{v}_{a}^{(k)}-v_{a}^{(k)}\right| \rightarrow 0
$$

as $k \rightarrow \infty$ for any link $a \in A$.

Theorem 5 simply states that the link tolls determined through the sequential pricing experiments converge to the first-best, marginal-cost pricing link tolls that give rise to the SO link flows, as the number of trials goes to infinity. Nevertheless, because the actual number of pricing trials could be very limited, one could end up with an inexact marginal-cost, or a second-best, pricing scheme. In this case, it is vital to determine the bound of the inefficiency or welfare loss of the current link toll pattern, thereby assessing whether or not further pricing experiments are needed.

The desirable inefficiency bound can be easily calculated using the bounding method developed in previous sections, with no more than the information needed for the pricing experiment itself. What are needed are the individual link cost functions, the current link toll 
charges and the easily available link flows observed after implementing the pricing scheme. Let $\left(u_{a}: a \in A\right)^{\mathrm{T}}$ be the current estimated toll charge, and 诤 $u$ ) be the observed link flow after implementing $\hat{u}$. Because the observed link flow pattern is in user equilibrium, then from eqn. (33), for each link $a \in A$, one can calculate

$$
\hat{\kappa}_{a}=\frac{\hat{u}_{a}}{\hat{v}_{a} t_{a}^{\prime}\left(\hat{v}_{a}\right)}, a \in A
$$

In the case of the BPR link cost function:

$$
t_{a}\left(v_{a}\right)=t_{a}^{0}\left(1+0.15\left(\frac{v_{a}}{C_{a}}\right)^{4}\right)
$$

where $t_{a}^{0}$ and $C_{a}$ are respectively the link free-flow travel time and link capacity, we have

$$
\hat{\kappa}_{a}=\frac{\hat{u}_{a}}{0.6 t_{a}^{0}}\left(\frac{C_{a}}{\hat{v}_{a}}\right)^{4}, a \in A
$$

From (35) with $p=4, \quad 0 \leq \hat{\kappa}_{a} \leq 1, a \in A_{1}$, we obtain

$$
\begin{aligned}
\gamma_{1}(\mathrm{BPR}, \hat{u}) & \leq \frac{(1+\text { 造 } p) p}{1+p}\left(\frac{\left(1+\kappa_{a} p\right)}{(1+p)}\right)^{\frac{1}{p}}-\hat{\kappa}_{a} p \\
& =\frac{\left(1+4 \mathrm{rec}_{a}\right) 4}{1+4}\left(\frac{\left(1+4 \kappa_{a}\right)}{(1+4)}\right)^{\frac{1}{4}}-4 \hat{\kappa}_{a} \\
& =4\left(\frac{1+4 \hat{\kappa}_{a}}{5}\right)^{\frac{5}{4}}-4 \hat{\kappa}_{a}
\end{aligned}
$$

From (39) with $p=4, \hat{\kappa}_{a} \geq 1, a \in A_{2}$, we obtain

$$
\begin{aligned}
\gamma_{2}(\mathrm{BPR}, \hat{u}) & \leq \kappa_{a}\left[\left(\frac{p}{1+p}\right)\left(\frac{1+\kappa_{a} p}{\kappa_{a} p}\right)\right]^{p+1}-1 \\
& =\kappa_{a}\left[\left(\frac{4}{1+4}\right)\left(\frac{1+4 \kappa_{a}}{4 \kappa_{a}}\right)\right]^{4+1}-1 \\
& =\kappa_{a}\left[\frac{4}{5}\left(\frac{1+4 \kappa_{a}}{4 \kappa_{a}}\right)\right]^{5}-1
\end{aligned}
$$

Note that, if the demand is fixed, we obtain the maximum inefficiency given by (28), with $\gamma_{1}$ and $\gamma_{2}$ provided by (87) and (88) and $\hat{\kappa}_{a}$ as in (86), based on the observed link flows. Then the estimated bound of the relative efficiency loss is followed by eqn. (7). If the demand is elastic, we have to use eqn. (62) to determine the maximum inefficiency with the above $\gamma_{1}$ and $\gamma_{2}$. The estimated bound of the relative efficiency loss is then readily calculated by eqn. (50). 
Example 5 We now illustrate application of the inefficiency bounding methods for the trial-and-error pricing scheme with unknown demand functions developed in Yang et al. (2004). Consider the same example used in Yang et al. (2004), with the network shown in Figure 7. The OD demand functions are given below:

$$
\begin{array}{ll}
D_{1 \rightarrow 7}\left(\mu_{1 \rightarrow 7}\right)=600 \exp \left(-0.04 \mu_{1 \rightarrow 7}\right) ; & D_{2 \rightarrow 7}\left(\mu_{2 \rightarrow 7}\right)=500 \exp \left(-0.03 \mu_{2 \rightarrow 7}\right) \\
D_{3 \rightarrow 7}\left(\mu_{3 \rightarrow 7}\right)=500 \exp \left(-0.05 \mu_{3 \rightarrow 7}\right) ; & D_{6 \rightarrow 7}\left(\mu_{6 \rightarrow 7}\right)=400 \exp \left(-0.05 \mu_{6 \rightarrow 7}\right)
\end{array}
$$

The BPR link cost function (85) is used with the input data given in Table 1.

Table 1. Input data for the network in Figure 7

\begin{tabular}{cccccccccccc}
\hline$a$ & 1 & 2 & 3 & 4 & 5 & 6 & 7 & 8 & 9 & 10 & 11 \\
$t_{a}^{0}$ & 6 & 5 & 6 & 7 & 6 & 1 & 5 & 10 & 11 & 11 & 15 \\
$C_{a}$ & 200 & 200 & 200 & 200 & 100 & 100 & 150 & 150 & 200 & 200 & 200 \\
\hline
\end{tabular}

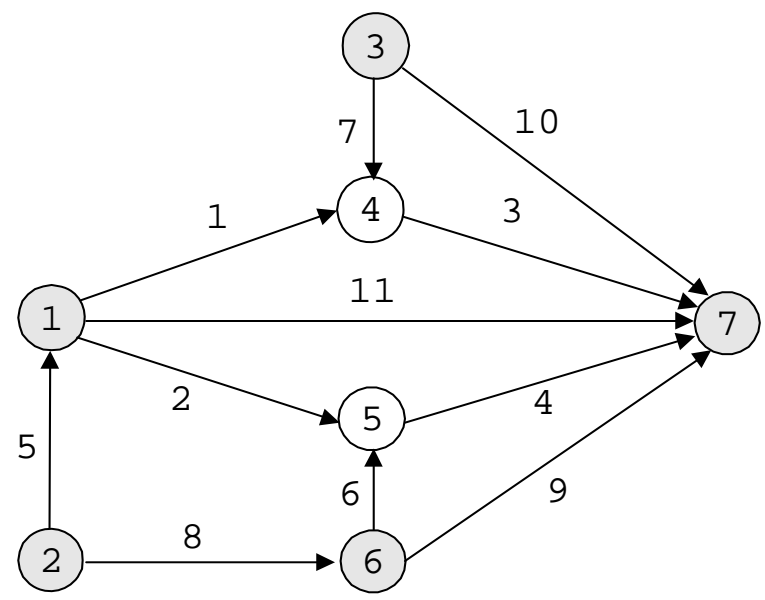

Figure 7. The network used for Example 5

We consider bounding the efficiency loss for the inexact marginal-cost pricing schemes in the sequential experimental pricing process for the elastic demand case with the above demand functions. The sequential pricing experiment starts with an initial un-tolled UE link flow pattern, using a sequence of $\alpha^{(k)}=1 /(k+1)$ that satisfies condition (84). Four levels of parameter omega are chosen: $\omega_{1}=\omega_{2}=1.5,2.0,2.5,3.0$ in the bounding formula (62).

The bounding results are shown in Figure 8, together with the actual efficiency loss. Note that the actual efficiency loss and the bound with the actual value of $\omega$ become known only 
if the demand functions are known and they are presented in the figure for comparative analysis. With appropriate a priori values of $\omega$, one can see that the bounds established with unknown demand functions are effective and indeed useful. It tells us whether a sufficiently good approximate marginal-cost pricing scheme is already found, without resorting to demand functions. Indeed, irrespective of the chosen value of parameter $\omega$, the actual and the maximum relative welfare gaps given by the formula all approach zero as the experiment continues and the tolls under trial reach the optimum, although the inter-medium bounds depend on its specific values. It is worthwhile to note that the bound with the actual $\omega$ is located in between the bounds for $\omega=1.5$ and $\omega=2.0$. This confirms our early observation that the practical $\omega$ value is within the range of $1.0 \leq \omega \leq 2.25$.

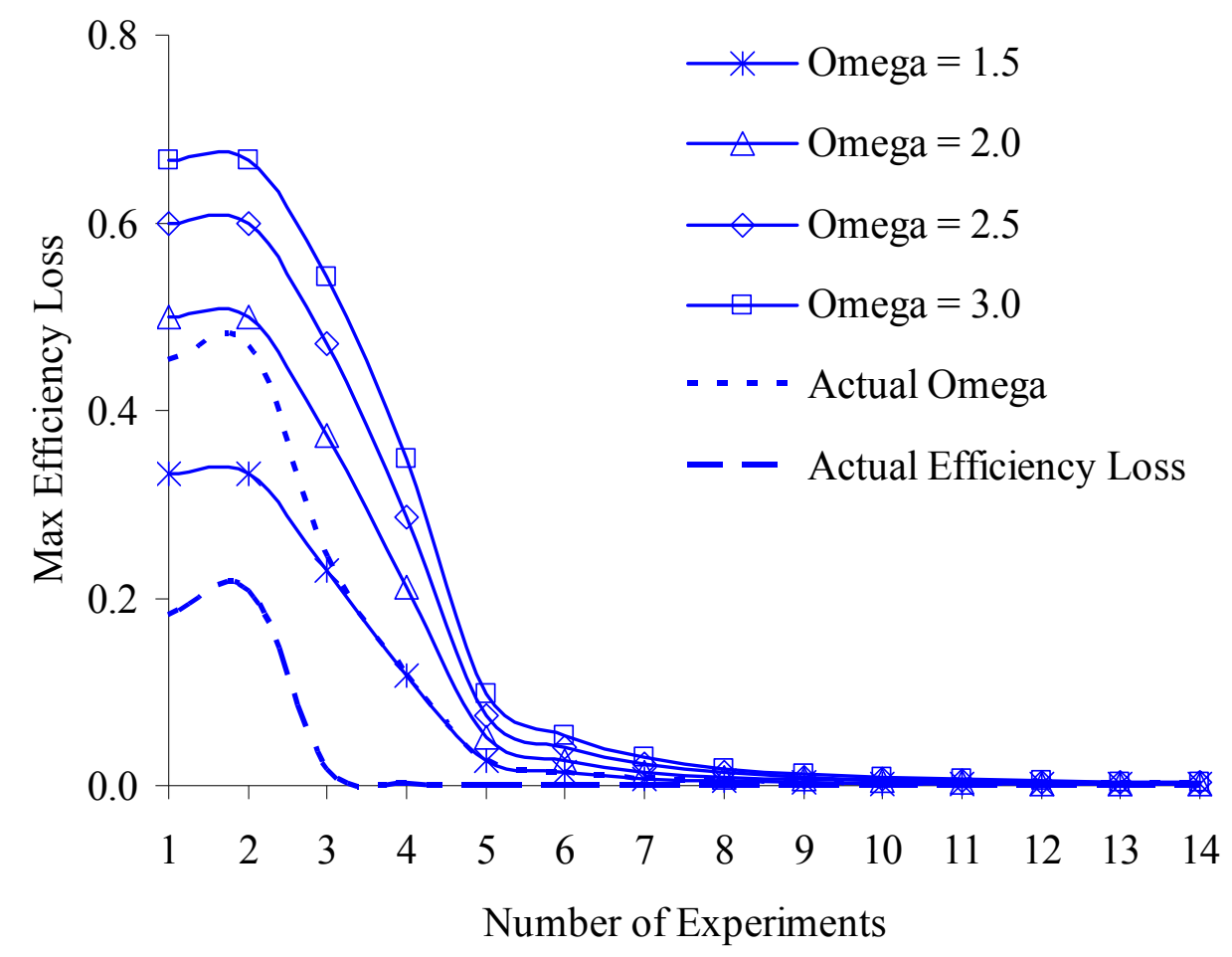

Figure 8. The upper bound of the relative efficiency loss in the sequential experimental pricing process with elastic demands

\section{Conclusions}

In this paper we examined the efficiency loss of a second-best congestion pricing scheme in general networks due to inexact marginal-cost pricing, with either fixed or elastic demand. We established the upper bound of the efficiency loss of a given congestion pricing scheme in the spirit of 'price of anarchy' that appeared in recent literature for bounding the inefficiency of equilibria in non-atomic congestion games. We show that our new bounding 
formula can generate the existing price of anarchy results for self-routing in the special case of zero pricing, and lead to zero efficiency loss or maximum efficiency gain in the special case of first-best marginal-cost pricing. In the general case of any given second-best pricing scheme, the inefficiency bound can be calculated with limited information. An application of the proposed inefficiency bounding methods is provided to assess the accuracy of inexact marginal-cost pricing in the sequential experimental pricing process with unknown demand functions developed previously.

We acknowledge that the method developed in this study is restricted to the standard traffic equilibrium problem, it can be further extended in a few manners: 1) to generalize the results to the case of non-separable and asymmetric link cost and OD demand functions (Perakis, $2004)$; 2) to extend the bounding method to the general traffic restraint and road pricing problem in capacitated networks (Yang and Bell, 1997; Correla et al., 2004); and 3) to bound the efficiency of second-best anonymous toll patterns for multiclass-user transportation networks (Yang and Huang, 2004).

\section{ACKNOWLEDGEMENT}

We thank an anonymous referee whose many insightful comments have significantly improved the presentation of the materials contained in this paper. We also acknowledge helpful discussions with Haijun Huang of Beijing University of Aeronautics and Astronautics, Xiaolei Guo and Zijia Tan of the Hong Kong University of Science and Technology. The research described here was substantially supported by a grant from the Research Grants Council of the Hong Kong Special Administrative Region, China (Project No. HKUST6215/06E).

\section{REFERENCES}

Beckmann, M.J., 1965. On optimal tolls for highways, tunnels and bridges. In: Vehicular Traffic Science (eds., Herman L.C. and Rothery R.). Elsevier, New York, pp.331-341.

Chau, C.K. and Sim, K.M., 2003. The price of anarchy for non-atomic congestion games with symmetric cost maps and elastic demands. Operations Research Letters 31, 327-334.

Correa, J.R., Schulz, A.S., and Stier-Moses, N.E., 2004. Selfish routing in capacitated networks. Mathematics of Operations Research 29, 961-976.

Correa, J.R., Schulz, A.S., and Stier Moses, N.E., 2005. On the inefficiency of equilibria in congestion games. Lecture Notes in Computer Science 3509, pp. 167-181, Springer.

Dafermos, S.C., 1980. Traffic equilibrium and variational ineqalities. Transportation Science 
$14,42-54$.

Dafermos, S.C., 1973. Toll patterns for multiclass-user transportation networks. Transportation Sciences 7, 211-223.

Dafermos, S.C. and Sparrow, F.T., 1971. Optimal resource allocation and toll patterns in user-optimized transport network. Journal of Transport Economics and Policy 5, 198200.

Goodwin, P.B., 1992. A review of new demand elasticities with special reference to short and long run effects of price changes. Journal of Transport Economics and Policy 26, 155169.

Lawphongpanich, S., Hearn, D.W. and Smith, M.J. (Eds.), 2006. Mathematical and Computational Models for Congestion Charging. Springer.

Oum, T.H., Waters, W.G. and Yong, J.S., 1992. Concepts of price elasticities of transport demand and recent empirical estimates. Journal of Transport Economics and Policy 26, 139-154.

Papadimitriou, C.H., 2001. Algorithms, games, and the internet. In: Proceedings of the $33^{\text {rd }}$ Annual ACM Symposium on the Theory of Computing, pp. 749-753.

Perakis, G., 2004. The "price of anarchy" under nonlinear and asymmetric costs. Lecture Notes in Computer Science 3064, pp. 46-58, Springer.

Pigou, A.C., 1920. The Economics of Welfare. MacMillan, London.

Roughgarden, T., 2005. Selfish Routing and the Price of Anarchy. The MIT Press, Cambridge, Massachusetts.

Roughgarden, T., 2003. The price of anarchy is independent of the network topology. Journal of Computer and System Sciences 67, 341-364.

Roughgarden, T. and Tardos, E., 2004. Bounding the inefficiency of equilibria in non-atomic congestion games. Games and Economic Behavior 47, 389-403.

Roughgarden, T. and Tardos, E., 2002. How bad is selfish routing? Journal of the ACM 49, 236-259.

Sheffi, Y., 1985. Urban Transportation Networks: Equilibrium Analysis with Mathematical Programming Methods. Prentice-Hall, Inc., Englewood Cliffs, NJ.

Smith, M.J., 1979a. The marginal cost taxation of a transportation network. Transportation Research 13B, 237-242.

Smith, M.J., 1979b. The existence, uniqueness and stability of traffic equilibrium. Transportation Research 13B, 295-304.

Vickrey, W.S., 1969. Congestion theory and transport investment. American Economic Review (Papers and Proceedings) 59, 251-261.

Walters, A.A., 1961. The theory and measurement of private and social cost of highway congestion. Econometrica 29, 676-699.

Wardrop, J.G., 1952. Some theoretical aspects of road traffic research. Proceedings of Institution of Civil Engineers-Part II, 1, 325-378. 
Yang, H. and Bell, M.G.H., 1997. Traffic restraint, road pricing and network equilibrium, Transportation Research 31B, 303-314.

Yang, H. and Huang, H.J., 2004. The multiclass, multicriteria traffic network equilibrium and system optimum problem. Transportation Research 38B, 1-15.

Yang, H. and Huang, H.J., 1998. Principle of marginal-cost pricing: How does it work in a general network? Transportation Research 32A, 45-54.

Yang, H. and Huang, H.J., 2005. Mathematical and Economic Theory of Road Pricing. Elsevier, Oxford.

Yang, H., Meng, Q. and Lee, D.H., 2004. Trial-and-error implementation of marginal-cost pricing on networks in the absence of demand functions. Transportation Research 38B, 477-493. 